\title{
A survey for nanodiamond features in the 3 micron spectra of Herbig Ae/Be stars ${ }^{\star} \star \star \star$
}

\author{
B. Acke ${ }^{1, \star \star \star}$ and M. E. van den Ancker ${ }^{2}$ \\ 1 Instituut voor Sterrenkunde, KULeuven, Celestijnenlaan 200B, 3001 Leuven, Belgium \\ e-mail: bram@ster.kuleuven.be \\ ${ }^{2}$ European Southern Observatory, Karl-Schwarzschild Strasse 2, 85748 Garching bei München, Germany
}

Received 16 May 2006 / Accepted 16 June 2006

\begin{abstract}
Aims. We have carried out a survey of $60 \mathrm{Herbig}$ Ae/Be stars in the 3 micron wavelength region in search for the rare spectral features at 3.43 and 3.53 micron. These features have been attributed to the presence of large, hot, hydrogen-terminated nanodiamonds. Only two Herbig Ae/Be stars, HD 97048 and Elias 3-1 are known to display both these features.

Methods. We have obtained medium-resolution spectra $(R \sim 2500)$ with the ESO near-IR instrument ISAAC in the $3.15-3.65$ micron range.

Results. In our sample, no new examples of sources with prominent nanodiamond features in their 3 micron spectra were discovered. Less than $4 \%$ of the Herbig targets show the prominent emission features at 3.43 and/or $3.53 \mu \mathrm{m}$. Both features are detected in our spectrum of HD 97048. We confirm the detection of the $3.53 \mu \mathrm{m}$ feature and the non-detection of the $3.43 \mu \mathrm{m}$ feature in MWC 297. Furthermore, we report tentative $3.53 \mu$ m detections in V921 Sco, HD 163296 and T CrA. The sources which display the nanodiamond features are not exceptional in the group of Herbig stars with respect to disk properties, stellar characteristics, or disk and stellar activity. Moreover, the nanodiamond sources are very different from each other in terms of these parameters. We do not find evidence for a recent supernova in the vicinity of any of the nanodiamond sources.

We have analyzed the PAH $3.3 \mu \mathrm{m}$ feature and the Pfund $\delta$ hydrogen emission line, two other spectral features which occur in the 3 micron wavelength range. We reinforce the conclusion of previous authors that flared-disk systems display significantly more PAH emission than self-shadowed-disk sources. The Pf $\delta$ line detection rate is higher in self-shadowed-disk sources than in the flareddisk systems.

Conclusions. We discuss the possible origin and paucity of the (nano)diamond features in Herbig stars. Different creation mechanisms have been proposed in the literature, amongst others in-situ and supernova-induced formation. Our data set is inconclusive in proving or disproving either formation mechanism.
\end{abstract}

Key words. circumstellar matter - stars: pre-main-sequence - planetary systems: protoplanetary disks

\section{Introduction}

Herbig Ae/Be (HAEBE) stars are pre-main-sequence objects of intermediate $\left(1.5-8 M_{\odot}\right)$ mass. These sources exhibit large infrared (IR) excesses, for the greater part due to thermal emission of circumstellar dust. The geometry of this dust surrounding the late-B, A and F-type members of this class is disklike (e.g. Mannings \& Sargent 1997; Testi et al. 2003; Piétu et al. 2003; Fuente et al. 2003; Natta et al. 2004). Also for early-B-type stars, evidence for disks has been found (e.g. Vink et al. 2002). However, there is growing evidence that the structure of the circumstellar matter in early-type B stars is fundamentally different from Herbig Ae and T Tauri stars (e.g. Fuente et al. 2003; Bik \& Thi 2004).

The chemistry and mineralogy of the dust component of circumstellar disks around HAEBE stars has been studied

* Based on observations collected at the European Southern Observatory, Paranal, Chile (program numbers 071.C-0653, 072.C-0648, 073.C-0245 and 074.C-0248).

$\star \star$ Figure 1, Table 4 and Appendix A are only available in electronic form at http: //www. edpsciences.org

$\star \star \star$ Postdoctoral researcher of the Research Fund KULeuven. with unprecedented precision based on near-to-mid-IR spectra (2-200 $\mu \mathrm{m}$ ) provided by the Infrared Space Observatory (ISO, Kessler et al. 1996). The launch of this satellite in 1995 was the start of a brand-new era for the research of protoplanetary disks. The spectra revealed a large variety in dust properties and species, from small carbonaceous molecules like polycyclic aromatic hydrocarbon molecules (PAHs) to silicate dust. Moreover, some sources were shown to contain crystalline grains, similar to those found in comets in our own solar system (Waelkens et al. 1996; Malfait et al. 1998a, 1999; van den Ancker et al. 2000a,b; Meeus et al. 2001). All available ISO 2-15 $\mu \mathrm{m}$ spectra of HAEBE stars have been studied as a whole by Acke \& van den Ancker (2004, hereafter AV04).

A handful of HAEBE sources (HD 97048 and Elias 3-1, Van Kerckhoven et al. 2002, and references therein) and the post-AGB binary HR 4049 (Geballe et al. 1989) display peculiar spectral features at 3.43 and $3.53 \mu \mathrm{m}$. Comparison with laboratory spectra has convincingly shown that the carriers of these features are hydrogen-terminated nanometer-sized diamonds (hereafter nanodiamonds, Guillois et al. 1999). Near-IR observations of HD 97048 by Habart et al. (2004b) have spatially resolved the emission region of the strong 3.43 and $3.53 \mu \mathrm{m}$ 
features on a scale of $0.18^{\prime \prime} \times 0.18^{\prime \prime}$. The observations prove beyond doubt that the emission region is confined to the inner $15 \mathrm{AU}$ of the circumstellar disk. More tentative detections of the features were reported in the literature (e.g. in HD 142527 and HD 100546, Waelkens et al. 1996; Malfait et al. 1998b, respectively), but only one additional $3.53 \mu \mathrm{m}$ feature was discovered persuasively (in MWC 297, Terada et al. 2001). Summing up, only two HAEBE stars display both the 3.43 and the $3.53 \mu \mathrm{m}$ feature, and one HAEBE object shows the $3.53 \mu \mathrm{m}$ feature. Considering that the ISO sample contains 45 targets, only a minor fraction of the HAEBE stars appear to have such a spectacular 3 micron spectrum (AV04).

The currently operating successor to the ISO satellite, Spitzer, is not equipped with a spectrograph which can observe in the 3 micron region of the spectrum. Ground-based observations are therefore the only alternative. With the present paper, we intend to enlarge the sample of HAEBE stars that are observed in the 3 micron region and possibly identify new targets which display nanodiamond emission. Therefore, we have employed the near-IR ISAAC instrument (ESO-VLT) and focused on the wavelength region around 3.3 (polycyclic aromatic hydrocarbons, hereafter PAHs), 3.43 and $3.53 \mu \mathrm{m}$ (nanodiamonds). In Sect. 2, we present the sample and the data reduction procedure. The detected features are discussed in Sect. 3.

\section{The data set}

\subsection{The sample stars}

The sample of HAEBE stars compiled for this survey has been based on Tables 1 and 2 in the paper of Thé et al. (1994) and on the list published by Malfait et al. (1998a). For 19 of the 60 observed targets, including HD 97048, spectroscopic data in the 3 micron region are present in the ISO data archive (AV04). The overlap between the two samples guarantees the continuity between the two data sets. This survey enlarges the number of high-quality 3 micron spectra of Herbig stars available to us from 45 (ISO) to 82 (ISO+new observations).

\subsection{Observations and data reduction}

The observations in the 3 micron region of the spectrum were performed using the medium-resolution spectroscopic mode of the ESO-VLT instrument ISAAC ${ }^{1}$, mounted on UT1 (ANTU). For each target two wavelength regions were recorded: the first is centered around 3.3 and the second around $3.5 \mu \mathrm{m}$. The wavelength coverage of each spectrum is about $0.25 \mu \mathrm{m}$. The width of the unresolved telluric lines in the spectra is a measure for the resolution of the spectra. This value is approximately $15 \AA$, corresponding to a spectral resolution of 2300. The spectra are oversampled with a pixel-to-pixel wavelength step of $2.5 \AA$. The width of the slit is $1^{\prime \prime}$, needed to achieve the medium spectral resolution. The sky background subtraction is done by chopping/nodding with a throw of $20^{\prime \prime}$ in the direction of the slit.

The 3 micron region is heavily affected by atmospheric absorption features. Standard stars (STDs) have been measured in order to correct for this effect. The straightforward data reduction procedure consists of a flatfield correction, wavelength calibration and multiplication with a responsivity curve. The latter is deduced from the STD spectrum, scaled to the right flux values using an appropriate Kurucz model. It was noted that the wavelength calibration of the target and standard star is never exactly

\footnotetext{
${ }^{1}$ http://www.eso.org/instruments/isaac/
}

the same. The offset, a fraction of a pixel up to a few pixels large, leads to artefacts in the resulting spectrum. We have applied a relative shift to the extracted STD spectrum to correct for this effect, in an attempt to reduce the residuals of atmospheric features.

During a few nights, no STD measurements were available to correct for the telluric line absorption. Some more nights, flatfield and/or wavelength calibration files were lacking ${ }^{2}$. In these cases, STD and calibration measurements from other nights were used. This increases the problem of wavelength calibration significantly, introducing second-order differences between the target and the STD spectrum. Furthermore, the assumption that the atmospheric conditions are approximately the same when observing target and standard star through the same airmass is not valid at all in these cases. We have tried to correct for the first order effect of this problem by applying an additional factor $(\sim 0.8-1.2)$ to the airmass ratio of target and standard star. This cosmetic surgery can of course not compensate for nightto-night differences in e.g. humidity.

The 3.3 and 3.5 micron spectra were reduced independently. When comparing the flux levels in the overlap region, fluxlevel jumps occur for a few sources. This is probably due to slit losses: the slit width is only a fraction of the atmospheric seeing. Variations in seeing between the observations of standard star and target make that a different fraction of the source's flux is captured. This effect translates to a multiplicative factor in the resulting spectrum. Other possible causes for the flux-level jumps include errors in background subtraction or differences in atmospheric conditions between the observations at 3.3 and 3.5 micron. We have corrected the jump between the two resulting spectra by applying a multiplicative factor to both spectra and scale them to the mean value in the overlap region. In most cases, this factor is of the order of $0.6-1.5$. We note that, since the PAH 3.3 micron and nanodiamond 3.53 micron feature are both included within one spectrum, their shape is insensitive to offsets in absolute flux level. The strength of these features is of course altered due to the application of the multiplicative factor.

Inherent to the reduction method, some residuals of telluric lines remain present in the final spectra. This is nevertheless not dramatic for our survey. Because of the medium spectral resolution of our ISAAC spectra $(\sim 2300)$, the telluric-line residuals are very narrow compared to the typical width $(F W H M \sim$ $0.03 \mu \mathrm{m})$ of the PAH and nanodiamond features. Furthermore a bright $3.53 \mu \mathrm{m}$ source similar to HD 97048 would be easily recognized, even without atmospheric corrections, due to the strength of the nanodiamond features.

The reduced ISAAC 3 micron spectra of the sample sources are presented in Fig. 1. We have rebinned the spectra to a spectral resolution of 750 for the sake of clarity. The broad PAH and diamond features remain clearly visible. The sharp, unresolved Pfund $\delta$ line on the other hand is spread out over 20 pixels and is therefore undetectable in most of the spectra shown in the figure. The smooth+rebin procedure makes that also the unresolved telluric-line residuals are spread out. In regions which are rich in telluric absorption lines, this may cause problems: the lines are overlapping each other and form absorption bands in the rebinned spectrum. This induces "absorption features". It is clear that, of the two wavelength regions, the $3.3 \mu \mathrm{m}$ spectra suffer the most from telluric line absorption. The region between 3.3

\footnotetext{
2 No STD in the $3.3 \mu \mathrm{m}$ region on $04-05 / 09 / 2003$ and $06-07 /$ $10 / 2004$; no STD in both the 3.3 and $3.5 \mu \mathrm{m}$ regions on $18-19 / 11 / 2003$ and 17-18/07/2004; no calibration files on $04-05 / 11 / 2003$ and 20-21/10/2004.
} 
and $3.32 \mu \mathrm{m}$ has been cut out. The measured flux in this region is close to zero in both science and standard-star observations, due to strong telluric absorption. Division by the STD spectrum induces a strong artefact in this wavelength interval. Note however that the PAH $3.3 \mu \mathrm{m}$ feature can still be discerned, since the onset and peak position of its spectral signature are located at shorter wavelengths.

When available, the ISO spectrum is overplotted in Fig. 1. The continuum flux levels of the ISAAC and ISO data are within a $30 \%$ error bar of the mean value of both for most targets. The experimental error bar on the ratio of the ISAAC continuum flux and the $L$-band magnitude - converted to the right units - is about $60 \%$. The latter indicates the poor absolute flux calibration quality of the ISAAC spectra.

\subsection{Characterizing the spectral features}

Spectral features that appear in the 3 micron spectra are in this analysis characterized with a few parameters. The integrated line flux (in $\mathrm{W} \mathrm{m}^{-2}$ ), full width at half maximum ( $F W H M$, in $\left.\mu \mathrm{m}\right)$, the peak flux (in Jy) and peak position (in $\mu \mathrm{m}$ ) are determined. We have estimated the statistical error on these measurements based on the noise on the spectra. The error on the line fluxes due to uncertainties on the absolute flux level calibration is estimated to be $60 \%$. This error is not included in the figures and tables presented in this paper. Note that the shape and the peakover-continuum ratio of the detected features is not affected by the absolute calibration of the spectra. In Sect. 3 we discuss the observed spectral features and include a comparison with the ISO measurements described in AV04.

\subsection{Classifying the spectral energy distributions}

We have classified the sample targets into the two Meeus et al. (2001) groups based on the shape of the spectral energy distribution (SED). This classification roughly represents the circumstellar disk's geometry: group I sources have a strong mid-IR excess which is believed to originate from a flared outer disk. Group II objects have a smaller mid-IR excess and non-flaring outer disks (Dullemond 2002). The sample targets in this paper are classified based in Fig. 2 (see also van Boekel et al. 2003; Dullemond et al. 2003; Acke et al. 2004, AV04). In this diagram, the ratio of near-IR and mid-IR luminosity is plotted versus the non-color-corrected IRAS [12]-[60] color. Group I sources and embedded objects end up in the lower right corner of the diagram, while group II sources have higher near-IR to mid-IR luminosity ratios.

Sources with an $A_{\mathrm{V}}$ value exceeding 4 have been classified as embedded sources. The link between SED shape and circumstellar geometry is less clear in these cases. In Fig. 2, we have also included another possible classification criterium. Almost all group I and all embedded sources have a 60 micron excess exceeding $10 \mathrm{mag}$. In group II most objects have an excess smaller than $10 \mathrm{mag}$. Both criteria - the diagram and the 60 micron excess - hence lead to more or less the same classification. In Table 5 we have indicated the classification based on the diagram.

Two sample stars, T CrA and T Ori, cannot be classified due to a lack of IR photometric measurements. However, both sources display photometric variability: their optical light curves are characterized by Algol-like minima, commonly referred to as UX Orionis (UXOR) behaviour. These minima are attributed to extinction events which are caused by dust and gas clumps

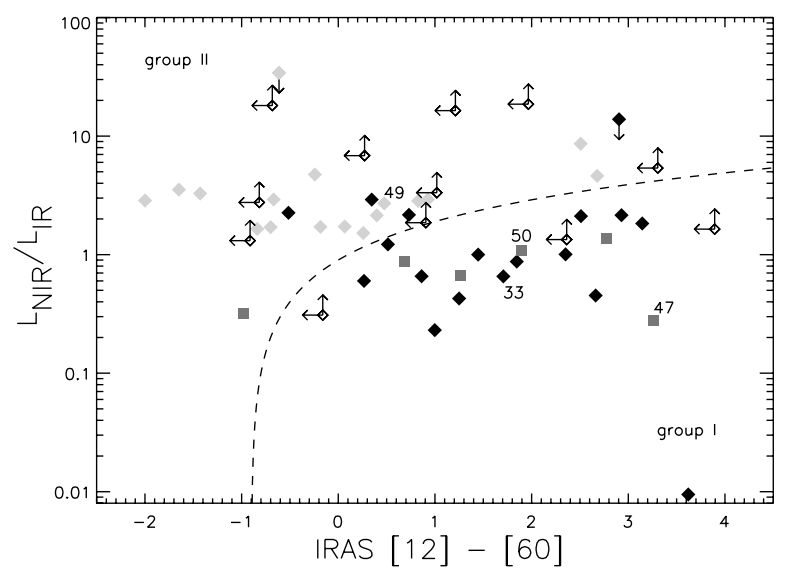

Fig. 2. The classification diagram of the sample targets. Sources with $A_{\mathrm{V}}$ values above 4 are definied to be embedded sources (grey squares). Objects on the left side of the empirical separation line (dashed line) are group II members, on the right side group I. A classification solely based on the 60 micron excess gives almost equal results. Objects with a 60 micron excess exceeding 10 mag (black diamonds) are almost all group I sources, while targets with an excess below 10 mag (grey diamonds) belong mostly to group II. Some targets have been marked with their sequence number (see Table 5); those are the (tentative) nanodiamond sources (see Sect. 3.4).

moving in and out the line of sight (Wenzel 1968; Grinin et al. 1988; Voshchinnikov 1990; Grinin et al. 1998). Dullemond et al. (2003) have argued that the clumps spring up from the puffed-up inner rim, and that all UXORs have self-shadowed disks. In other words, all UXOR stars should be group II members. The authors indeed find this correlation in a large sample of Herbig stars. Based on this conclusion, we classify the UXOR sample targets $\mathrm{T} \mathrm{CrA}$ and T Ori as group II members.

In the present sample, 15 sources have been classified as group I members, 32 as group II sources and 6 as embedded objects. 7 sources remain unclassified due to a lack of photometric measurements and/or upper limits.

\section{Description of the detected features}

In Table 5 we summarize the detection of features in the 3 micron spectra of the sample stars. A detection is defined as being a measurement of a spectral feature with a line flux exceeding $3 \sigma$. Tentative detections are features with line fluxes between 1 and $3 \sigma$. We have searched for the narrow Pfund $\delta$ line at $3.296 \mu \mathrm{m}$, the PAH $3.3 \mu \mathrm{m}$ feature and the nanodiamond features at 3.43 and $3.53 \mu \mathrm{m}$. In five sources, also the Humphreys series of the hydrogen atom was detected.

\subsection{The Pfund $\delta$ emission line}

The Pfund $\delta$ line at $3.296 \mu \mathrm{m}$ is located at the longer wavelength end of a region which is rich in telluric lines. The line is spectrally unresolved in all cases (i.e. $F W H M$ of the order of $15 \AA$ or equivalently $130 \mathrm{~km} \mathrm{~s}^{-1}$ ). We have determined the characterizing parameters of the $\operatorname{Pf} \delta$ line at the full resolution of the ISAAC spectra $(R=2300)$. The error bars on the line flux are consequently rather large. They are calculated from the noise in the 3.3 micron region of the reduced spectrum. The error includes the photon noise, as well as the noise induced by telluric line residuals. The latter is by far more important.

In the ISAAC sample of 60 sources, we detected the $\operatorname{Pf} \delta$ emission line in 16 targets or $27 \%$. Four more objects 

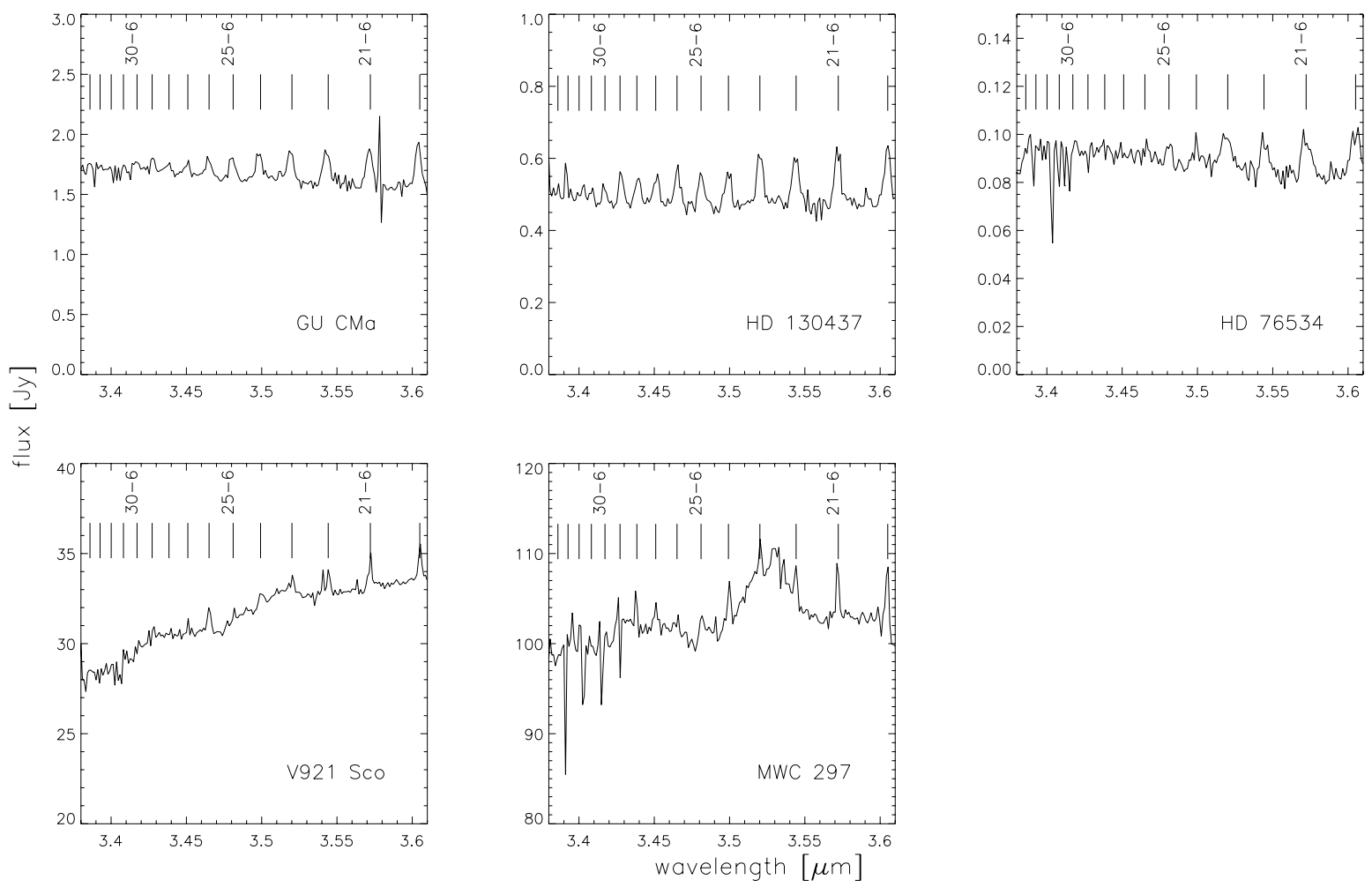

Fig. 3. The ISAAC 3.5 micron spectra of GU CMa, HD 76534, HD 130437, V921 Sco and MWC 297. The Humphreys series of the HI atom are indicated with vertical lines. The spectra in the top panels are clearly different from the spectra in the bottom panels. The first 3 targets are most likely normal Be stars, with the 3 micron continuum flux emanating from the stellar photosphere. The latter two are early-type accretion-disk systems. The near-IR continuum is due to hot circumstellar dust.

tentatively display this feature, which adds up to a detection rate of $33 \%$ in this sample.

The Pfund $\delta$ line has been (tentatively) detected in only three group I sources (HD 259431, HD 100546 and HD 142527). The detection rate in this group hence is $13-20 \%$. In group II, $10-13$ sources (tentatively) display the feature $(31-41 \%)$, while half of the six embedded sample stars show the emission line $(50 \%)$. In the group of unclassified sample stars, the detection rate is $14 \%$. The Pfund $\delta$ line is more detected in group II and in the embedded sources, than in group I. This difference is not statistically significant on a $5 \%$ level.

\subsection{The Humphreys series}

Five of the sample stars (8\%) show the Humphreys series of the HI atom in emission: GU CMa, HD 76534, HD 130437, V921 Sco and MWC 297 (for the latter, see also Terada et al. 2001). These objects are all early-type stars (O8-B2). The SED of GU CMa, HD 76534 and HD 130437 does not show a significant near-IR excess. This indicates that these sources are probably normal Be stars, rather than Herbig Be stars. The SEDs of V921 Sco and MWC 297 on the other hand are dominated by thermal emission of the circumstellar dust. The extinction in the line of sight towards these targets is strong, $A_{\mathrm{V}}=5.1$ and 7.8 respectively. These massive stars are most likely surrounded by accretion disks. In Fig. 3, the 3.5 micron spectra of GU CMa, HD 76534, HD 130437, V921 Sco and MWC 297 are presented. The Humphreys series is clearly visible.

The presence of the hydrogen Humphreys series in the 3 micron spectra correlates well with the presence of the hydrogen Pfund $\delta$ line: the $\operatorname{Pf} \delta$ line is detected in the spectra of GU CMa, HD 130437, V921 Sco and MWC 297. The 3.3 micron spectrum of HD 76534 is rather noisy. We have not detected an emission line at $3.296 \mu \mathrm{m}$ exceeding the $1 \sigma$ level in the latter case.

\subsection{The PAH 3.3 micron feature}

The $3.3 \mu \mathrm{m}$ feature, attributed to the streching vibrations of the $\mathrm{CH}$ bond in $\mathrm{PAH}$ molecules, is detected in 7 Herbig sources $(12 \%)$. Four additional sources tentatively show the feature. This suggests an upper limit for the detection rate of $18 \%$ in the ISAAC sample. The emission band has previously been detected in the infrared spectra of sample stars HD 97048, HD 100546 and HD 179218 (see e.g. AV04). We report new detections for HD 34282, V599 Ori and AS 310. Tentative detections are obtained for V346 Ori, V590 Mon, HD 101412 and HBC 687. Also the ISAAC and ISO spectra of TY CrA contain the 3.3 micron feature. Nevertheless, this source is most likely not a HAEBE star, considering the absence of $\mathrm{H} \alpha$ emission and the fact that the IR emission emanates from the "TY CrA bar" which is not directly associated to the object (Siebenmorgen et al. 2000). For HD 34282, we have previously reported a non-detection of the PAH 3.3 micron feature in the ISO spectrum (AV04). The upper limit on the line flux deduced from the ISO spectrum is $28.1 \times 10^{-16} \mathrm{~W} \mathrm{~m}^{-2}$, which is consistent with the current detection in the ISAAC spectrum $\left(24 \pm 7 \times 10^{-16} \mathrm{~W} \mathrm{~m}^{-2}\right)$. The detections of the PAH feature in the ISO spectra of HD 142666, HD 142527, V921 Sco and MWC 297 are not confirmed in the present spectra. For HD 142527 and MWC 297, strong Pf $\delta$ emission is observed, which is probably misinterpreted as $\mathrm{PAH}$ emission in the lower spectral resolution - ISO spectra. Also in V921 Sco (=CD-42 11721$)$, strong $\operatorname{Pf} \delta$ emission is detected. The 


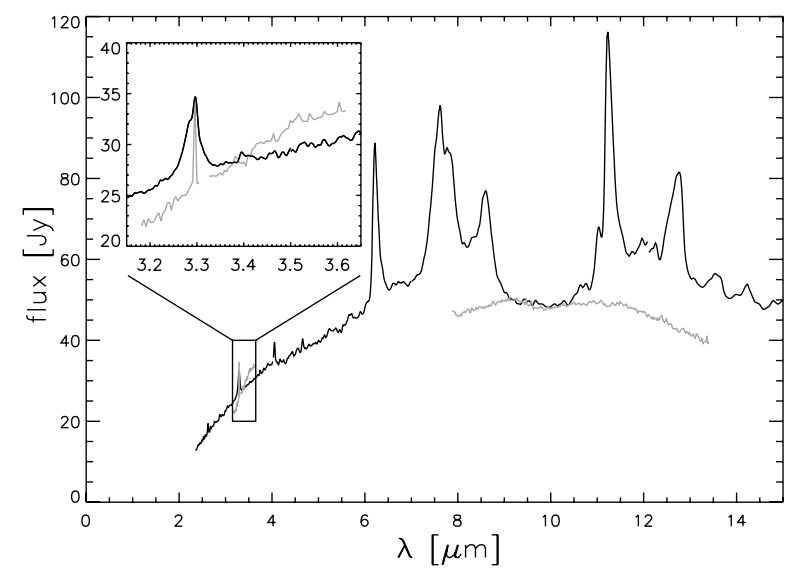

Fig. 4. The infrared spectrum of V921 Sco (CD-41 11721$)$. The black curve is the ISO spectrum $(R=150)$. The grey curve around 10 micron is the long-slit TIMMI2 spectrum (courtesy of R. van Boekel). This spectrum is scaled to the IRAS 12 micron flux. For clarity, a blow-up of the 3 micron wavelength region is shown in the inset. The grey curve in this panel is the ISAAC spectrum $(R=750$, not scaled); the black curve is the ISO spectrum at a resolution of 500. The slope of the ISAAC spectrum clearly deviates from the slope of the ISO spectrum at 3 micron, most likely due to slit losses. The ISO spectrum is dominated by strong PAH features. No PAH emission is detected in the ground-based spectra. The ISAAC spectrum shows the Pfund $\delta$ line, the Humphreys series and possibly a $3.5 \mu \mathrm{m}$ feature. In the high-resolution ISO spectrum, the $\operatorname{Pf} \delta$ line is visible on top of the PAH $3.3 \mu \mathrm{m}$ feature. The TIMMI2 spectrum reveals that beneath the overwhelming PAH emission in the ISO spectrum, a shallow silicate absorption band resides.

measured line flux in the ISAAC spectrum is however not sufficient to explain the measured ISO $3.3 \mu \mathrm{m}$ line flux. Remarkably, also in the ground-based TIMMI $2^{3}$ slit spectrum of V921 Sco in the 10 micron wavelength region, no PAH features are detected (Fig. 4). This may be surprizing, since the ISO spectrum of this source is dominated by strong PAH emission. The explanation is found in the extent of the emission region. Because of the large aperture size $\left(14^{\prime \prime} \times 20^{\prime \prime}\right)$, the ISO-SWS instrument has picked up PAH emission from the wider surroundings of the targets. Slit spectroscopy only selects a much narrower region around the central source. For V921 Sco, this remark is in agreement with the result of AV04. Table 9 in that paper reveals that the luminosity of the observed PAH emission in this source roughly corresponds to what one may expect from a tenuous halo. No emission features are detected in the 3.3 micron region of the ISAAC spectrum of HD 142666, possibly indicating that also in this case, the PAH emission is extended.

In Fig. 5 we have compared the peak-over-continuum ratio of the PAH 3.3 micron feature measured in the ISO and ISAAC spectrum. The measurements/upper limits of HD 34282 (\#2), HD 100546 (\#34) and HD 179218 (\#58) agree within the error bars. For HD 142527 (\#43) and MWC 297 (\#50), strong Pfund $\delta$ emission led to a false PAH $3.3 \mu \mathrm{m}$ detection in the ISO spectrum. In the previous paragraph we argued that the PAH emission in HD 142666 (\#42) and V921 Sco (\#47) is likely extended, and therefore not detected in the ISAAC spectrum. The ISO spectra of HD 97048 show significantly stronger PAH 3.3, NANO 3.4 and NANO $3.5 \mu \mathrm{m}$ features than the ISAAC spectrum. The relative ratios between the ISO and ISAAC line fluxes are $2.9 \pm 0.7,2.4 \pm 0.2$ and $1.65 \pm 0.02$ respectively. The ISO aperture is many times larger than the slit width of ISAAC. The PAH and nanodiamond emission emanates

${ }^{3}$ http://www.ls.eso.org/lasilla/sciops/3p6/timmi/

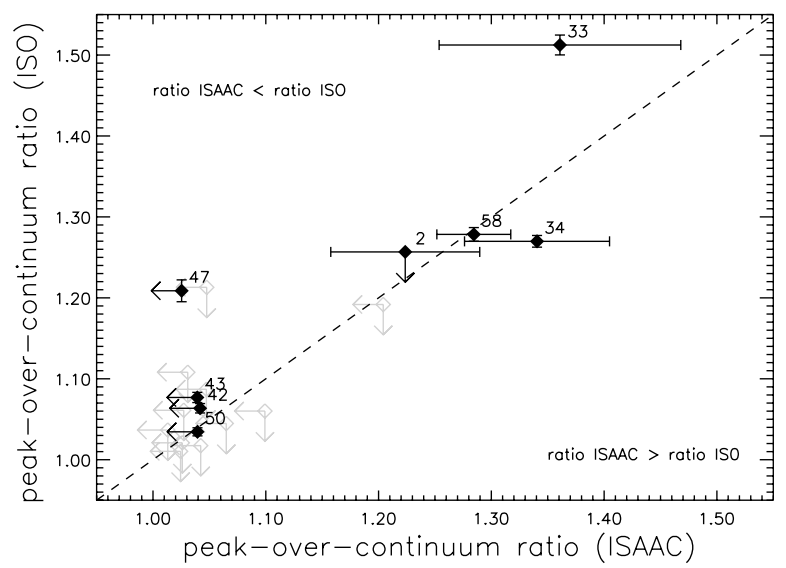

Fig. 5. Comparison of the peak-over-continuum ratio of the PAH $3.3 \mu \mathrm{m}$ feature, measured in the ISO and ISAAC spectrum. Filled symbols indicate targets for which we have detected the feature in at least one of the infrared spectra. The numbers refer to the target numbers listed in Table 5. Upper limits are indicated by arrows. The dashed line represents ratio ISAAC $=$ ratio ISO.

from the circumstellar disk's surface. The estimated extent is of the order of a few tens of an arcsecond (van Boekel et al. 2004; Habart et al. 2004a,b). This extent, smeared out by the atmospheric seeing, could lead to a loss of PAH and NANO emission in the ISAAC spectrum, relative to the ISO spectrum. The underlying continuum flux, which originates from the very inner parts of the disk, is less sensitive to this effect. This potentially explains the weakness of the features in the ISAAC spectrum. Alternatively, the PAH and NANO features might display temporal variability. This variability has not been noted in the five ISO 3 micron spectra which are available for this source, however. These ISO observations span about one year and a half (AV04).

The ISAAC detection rate of the PAH $3.3 \mu \mathrm{m}$ feature in the group I sources is $36 \%$ (50\% including tentative detections). The rate in group II is much lower (0-3\% respectively). A PAH $3.3 \mu \mathrm{m}$ feature was detected in the spectrum of the embedded source V599 Ori (17\%). In the computation of these numbers, we have excluded GU CMa, HD 76534, HD 130437 and TY CrA, which are most likely no Herbig stars. The high and very low detection rate in respectively group I and II confirms the main conclusion of AV04. The PAH features emanate from the surface of a flared circumstellar disk. Only group I sources have a flared disk and are expected to display strong PAH features (Habart et al. 2004a, AV04). In Table 1 we compare the detection rates of the PAH 3.3 micron feature in the ISO sample to the rates in the present ISAAC sample. The ISO sample contains 16 group I, 22 group II and 7 embedded sources ${ }^{4}$. The combined ISO+ISAAC sample consists of 25 group I, 42 group II, 11 embedded and 4 unclassified targets, in total 82 sources. A number of targets are present in both the ISO and ISAAC sample. The sources with a PAH detection in the ISO spectrum but not in the ISAAC spectrum, or vice versa, were all discussed in the previous paragraphs. We have consistently included these results in the computation of the detection rates. Up to half of the sources classified in group I display the PAH $3.3 \mu \mathrm{m}$ feature, while it is detected in only a small number of group II and embedded objects.

4 We have classified T CrA as group II member and V921 Sco as embedded source in stead of in group I (as was done by AV04). 
Table 1. The detection rates of the PAH $3.3 \mu \mathrm{m}$ feature in the ISO and ISAAC samples. T stands for the total of classified sample sources, I and II refer to the Meeus groups and E represents the embedded sources. The last row gives the detection rates in the combined ISO+ISAAC sample. When two percentages are given, the second refers to the detection rate including tentative detections. The detection rate in group I is significantly higher than in group II (confidence level $>99.5 \%$.

\begin{tabular}{r|cccc}
\hline \hline & $\mathrm{T}$ & $\mathrm{I}$ & $\mathrm{II}$ & $\mathrm{E}$ \\
\hline ISO & $22-29 \%$ & $44-56 \%$ & $14 \%$ & $0-14 \%$ \\
ISAAC & $12-18 \%$ & $36-50 \%$ & $0-3 \%$ & $17 \%$ \\
total & $14-21 \%$ & $32-48 \%$ & $5-7 \%$ & $9 \%$ \\
\hline
\end{tabular}

According to AV04, the PAH 3.3 micron feature is the least detected PAH feature in the 3-11 micron region in the ISO sample. It is present in only $44 \%$ of the cases where the 6.2 micron feature is detected. The latter is the most detected PAH feature in the ISO study. Furthermore, the PAH 6.2 feature is always detected when the PAH $3.3 \mu \mathrm{m}$ feature is present ${ }^{5}$. Using Bayes's theorem ${ }^{6}$, one can estimate the hypothetical detection probability of the 6.2 micron feature in the total sample. We assume that the detection probability of the 3.3 micron feature is equal to the relative detection rate in the total sample. The conditional probabilities - the relative detection rate of one feature, given the presence of the other - are taken from the ISO study (AV04). For group I, the hypothetical PAH 6.2 micron detection probability is $73 \%-100 \%$, while it is $11 \%-16 \%$ for group II and $20 \%$ for the embedded sources respectively. Hypothetically, three quarters to all flared disk sources are PAH emitters. Most of the PAH detections in group II and in the embedded sources, as well as a fraction of the group I detections, are likely due to extended emission from a circumsystemic nebula.

\subsection{The nanodiamond features}

It is clear that our ISAAC sample does not contain any targets with a high $3.53 \mu \mathrm{m}$ feature/continuum ratio except HD 97048. This in itself is an important result. The detection of a strong $3.53 \mu \mathrm{m}$ feature in the near-IR spectra of Herbig stars is thus limited to Elias 3-1, HD 97048 and MWC 297. This leads to a detection rate of $3 / 82=4 \%$ for the entire ISO+ISAAC sample. The $3.43 \mu \mathrm{m}$ feature is even more rare, since it has been detected in Elias 3-1 and HD 97048 only (2\% of the sample).

We confirm the detection of the 3.53 micron feature in MWC 297 (Terada et al. 2001, AV04). The shape of this complex band resembles that of the $3.53 \mu \mathrm{m}$ feature in HD 97048, in the sense that the red wing is steeper than the blue wing and the peak position is around $3.529 \pm 0.002 \mu \mathrm{m}$. It is therefore likely that these features have a common carrier, i.e. (nano)diamonds. The line flux ratio of the 3.43 and $3.53 \mu \mathrm{m}$ features is 0.82 (ISO) for Elias 3-1 and 0.41 (ISAAC; ISO: 0.59) for HD 97048. The upper limit to this ratio is 0.39 (ISO; ISAAC: 2.0) for MWC 297. This indicates that the NANO $3.43 \mu \mathrm{m}$ is, if present, at least weaker than in the two other (nano)diamond sources.

A few more sample targets display a feature around $3.5 \mu \mathrm{m}$. In the Appendix, we argue that most of these features are artefacts. Only for V921 Sco, HD 163296 and T CrA, a potentially

${ }^{5}$ Except for HD 142527, a PAH $3.3 \mu$ m detection which was however not confirmed by the ISAAC spectrum.

${ }^{6} P(B)=\frac{P(B \mid A) \cdot P(A)}{P(A \mid B)}$ with $\mathrm{A}$ and $\mathrm{B}$ stochastic events. In this case A and $\mathrm{B}$ represent the detection of the 3.3 and the $6.2 \mu \mathrm{m}$ feature in the spectrum, respectively. real feature is detected. These detections are by far not sure, however. In the following, we refer to these sources as the candidate nanodiamond emitters.

\section{What makes the nanodiamond emitting sources so special?}

It is clear that very few targets display nanodiamond features in their 3 micron spectra. The main question which is addressed in this paper concerns the origin of these features. In this section, we will show that the 3.43 and $3.53 \mu \mathrm{m}$ emitting sources are no outliers in the group of Herbig stars as a whole (1), but also that these targets have very different stellar and circumstellar parameters compared to each other (2).

\subsection{Nanodiamonds and polycyclic aromatic hydrocarbons}

PAHs and nanodiamonds are both carbonaceous grains. The 3.3, 3.43 and 3.53 features have been attributed to the $\mathrm{C}-\mathrm{H}$ bonds in these structures. The emission of both species has been shown to be spatially extended and linked to the surface of the circumstellar disk (van Boekel et al. 2004; Habart et al. 2004a,b, AV04). Figure 6 displays the PAH $3.3 \mu \mathrm{m}$ line flux versus the nanodiamond $3.53 \mu \mathrm{m}$ line flux. The line fluxes determined for Elias 3-1 are included as well. The latter are based on the ISO spectrum of this source, rebinned to a resolution of 500. For the sake of clarity, we left out the upper limits when both features were undetected. Although a correlation between PAHs and nanodiamonds may be expected based on their common chemical root, the PAH $3.3 \mu \mathrm{m}$ feature is not detected in MWC 297 and the three tentative nanodiamond emitters V921 Sco, HD 163296 and T CrA. Furthermore, the PAH $3.3 \mu \mathrm{m}$ feature is obviously much more common than the nanodiamond features in the group of HAEBEs. There does not seem to be a connection between them. However, it should be noted that the line flux ratio of PAH $3.3 \mu \mathrm{m}$ and NANO $3.53 \mu \mathrm{m}$ in HD 97048 and Elias 3-1 the only two sources where both features are detected - is significantly smaller than $1(0.13 \pm 0.03$ and $0.5 \pm 0.1$, respectively $)$. The upper limit for this ratio in the other four $(1+3$ candidate $)$ nanodiamond targets is larger than unity. Hence it may be that the PAH $3.3 \mu \mathrm{m}$ feature is present, but too weak to be detected in the latter sources. If it is always true that the line flux ratio of PAH $3.3 \mu \mathrm{m}$ and NANO $3.53 \mu \mathrm{m}$ is smaller than one, the upper limits on the NANO 3.5 line flux in the other stars with detected PAH emission indicate that no nanodiamond feature can be present; the lower limits on the line flux ratios are all above one in these targets. In conclusion, the nanodiamond feature at $3.53 \mu \mathrm{m}$ appears to be stronger than the PAH $3.3 \mu \mathrm{m}$ feature. In the other sense, the presence of a PAH $3.3 \mu \mathrm{m}$ feature does not imply the presence/absence of a nanodiamond feature.

\subsection{Appearance of the disk}

As mentioned before, the (nano)diamond features are linked to the circumstellar disk. Here we investigate whether the disks of MWC 297, Elias 3-1 and HD 97048 are in any way different from other HAEBE disks. We have summarized some overall disk parameters of the (candidate) nanodiamond sources in Table 2. As a reference, we list the median value of each parameter in each group. The median values of $L_{\mathrm{exc}} / L_{\star}$ and the spectral index (a proxy for dust particle size) are not significantly different in sources with nanodiamond features as opposed to the sources without. 


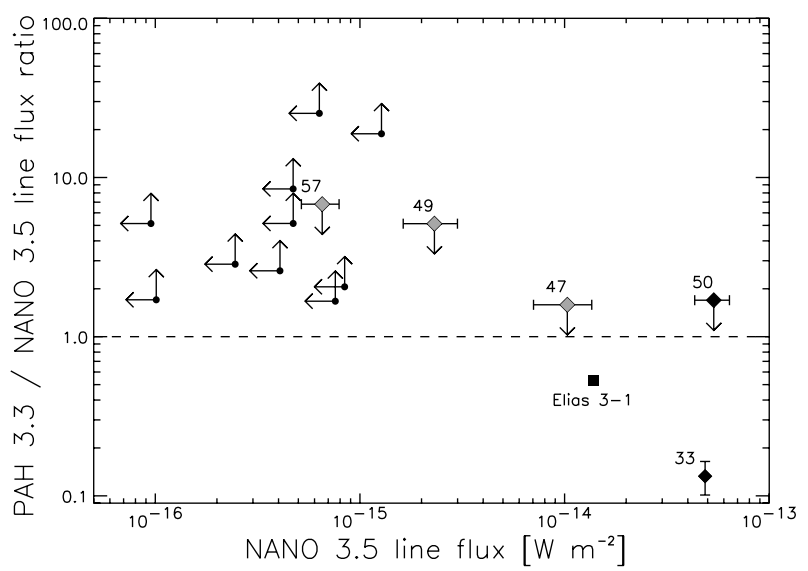

Fig. 6. The line flux ratio of the PAH $3.3 \mu \mathrm{m}$ and the nanodiamond $3.53 \mu \mathrm{m}$ feature versus the NANO $3.53 \mu \mathrm{m}$ line flux. The dashed line indicates where both line fluxes are equal. The black square indicates the ISO measurements of Elias 3-1. The black diamonds represent HD 97048 and MWC 297. The grey diamonds are the nanodiamond candidates V921 Sco, HD 163296 and T CrA. The black dots represent the targets with a detected PAH 3.3, and undetected NANO 3.53 feature. Only in HD 97048 and Elias 3-1, both features are detected. The line flux ratio of the PAH 3.3 and NANO 3.53 features is smaller than one in these targets, while the upper limits on this ratio are larger than unity in the other (candidate) nanodiamond emitters.

Table 2. Some disk parameters of the (candidate) nanodiamond sources. The Meeus group indicates the overall geometry of the disk. The ratio of the total IR excess to the stellar luminosity, $L_{\text {exc }} / L_{\star}$, indicates the fraction of stellar flux that is captured and reprocessed by the disk. For passive disks, this number represents the fraction of the sky covered by the disk, as seen from the central star. The ratio for T CrA is likely overestimated due to strong nearby IR emission, not directly linked to the system. The parameter $n$ is the spectral index in the $450 \mu \mathrm{m}-1.3 \mathrm{~mm}$ range, defined by $\lambda F_{\lambda} \propto \lambda^{-n}$.

\begin{tabular}{clccc}
\hline \hline$\#$ & Target & Group & $L_{\text {exc }} / L_{\star}$ & $n$ \\
\hline & Elias 3-1 & I & $0.5-1.3^{a}$ & $3.23 \pm 0.03$ \\
33 & HD 97048 & I & 0.40 & $>2.9$ \\
50 & MWC 297 & E & 0.06 & $3.1 \pm 0.2$ \\
\hline 47 & V921 Sco & E & 0.11 & $4.1 \pm 0.3$ \\
49 & HD 163296 & II & 0.34 & $2.9 \pm 0.1$ \\
57 & T CrA & II? & 3.8 & $?$ \\
\hline & Median & I & 0.50 & 3.9 \\
& Median & II & 0.44 & 3.0 \\
& Median & E & 0.63 & 4.1 \\
\hline
\end{tabular}

${ }^{a}$ For Elias 3-1 we used a stellar luminosity of 20-50 $L_{\odot}$ (see Table 3).

\subsection{Stellar characteristics}

MWC 297, Elias 3-1, HD 97048 and the three candidate nanodiamond stars have very different stellar parameters. We have summarized their spectral type, estimated age and stellar luminosity in Table 3 . The median parameters for the total sample are again given as a reference. Nanodiamond features are found in sources with a wide range of spectral types (B1.5-F0). Although the nanodiamond sources might be a bit older ( $>1 \mathrm{Myr})$ than typical for Herbig stars, there exists no strong correlation with age; there are many examples of Herbig stars with similar ages in our sample which do not show nanodiamond features of comparable strength.

The absolute strength of the nanodiamond features (i.e., the nanodiamond luminosity, included in Table 3) appears to be linked to the spectral type (and hence $T_{\text {eff }}$ and luminosity) of
Table 3. Some stellar parameters of the (candidate) nanodiamond targets, and the observed nanodiamond $3.53 \mu \mathrm{m}$ luminosity $L_{3.5}$. The median value of each stelllar parameter in the total sample is given as a reference. Values taken from Houk \& Cowley (1975); Finkenzeller \& Mundt (1984); Shore et al. (1990); van den Ancker et al. (1998); Mora et al. (2001); Fuente et al. (2001); Habart et al. (2003); Acke et al. (2004); Hamaguchi et al. (2005), AV04 and references therein.

\begin{tabular}{llcccc}
\hline \hline$\#$ & Target & Sp. Type & $\log$ Age/yr & $\log L_{\star} / L_{\odot}$ & $L_{3.5} / L_{\odot}$ \\
\hline & Elias 3-1 & A6:e & $6.3-6.9$ & $1.3-1.7$ & $1 \times 10^{-2}$ \\
33 & HD 97048 & B9.5Ve+sh & $>6.3$ & 1.9 & $5 \times 10^{-2}$ \\
50 & MWC 297 & B1.5Ve & $5-6$ & 4.2 & $1 \times 10^{-1}$ \\
\hline 47 & V921 Sco & B0IVep & $5-6$ & 4.9 & $8 \times 10^{-2}$ \\
49 & HD 163296 & A3Ve & 6.6 & 1.6 & $1 \times 10^{-3}$ \\
57 & T CrA & F0e & $?$ & 0.8 & $3 \times 10^{-4}$ \\
\hline & Median & A2 & 6.0 & 1.7 & \\
\hline
\end{tabular}

the central star. This weak correlation appears to be the only one which can be derived. The nanodiamonds in the circumstellar disk must be heated to a certain temperature to be able to radiate in the near-IR. Hotter stars heat larger volumes of circumstellar dust. The extent of the nanodiamond emission region is therefore expected to be larger around earlier-type stars. This is in agreement with the observed correlation.

One of the current hypotheses on the presence of the nanodiamonds in our own solar system suggests that they were formed in a supernova outflow, and injected into the system (idea originally put forward by Clayton 1989, see Sect. 5). Under this hypothesis, the presolar nebula is enriched in matter from the supernova outflow. The parent star of a disk which contains supernova-injected diamonds must therefore be polluted by supernova material as well. Supernova outflows harbor $r$ - and $p$-process elements and specific isotopes of lighter elements. Enrichment of the stellar photosphere by such an outflow is expected to alter the photospheric abundance pattern of the star significantly. An abundance analysis of the nanodiamond sources opposed to stars with comparable stellar parameters might clarify whether or not supernovae are indispensable for the formation of (nano)diamonds in their circumstellar disks. For HD 97048, two high-quality optical spectra are available to us: a FEROS 3900-9100 A spectrum with a signal-to-noise ratio $(\mathrm{S} / \mathrm{N})$ of 115 , and a UVES $4800-6800 \AA$ spectrum with a $\mathrm{S} / \mathrm{N}$ of 460 . The latter spectrum is compiled from 12 UVES spectra, obtained in a time span of a few days. The spectra have been previously published in Acke \& Waelkens (2004) and Acke \& van den Ancker (2006), respectively. Based on the Vienna Atomic Line Database (VALD, Kupka et al. 1999), we have looked for the strongest absorption lines of elements with atomic number larger than $30(\mathrm{Zn})$. At none of the VALD wavelengths, an absorption line is detected. We have deduced upper limits on the equivalent width of the strongest lines and hence on the abundances of four heavy elements. The abundances of $\mathrm{Y}, \mathrm{Zr}$ and $\mathrm{Ba}$ in HD 97048 are less than a few times the solar abundance. Strontium is even depleted by at least a factor 10 compared to our sun. We conclude that there is no observational evidence for a photosphere enriched with heavy elements in the nanodiamond source HD 97048, and hence no evidence for the occurance of a recent supernova event in the target's proximity.

\subsection{Disk and stellar activity}

In Fig. 7, the line flux ratio of the $\operatorname{Pf} \delta$ line and the nanodiamond $3.53 \mu \mathrm{m}$ feature is plotted versus the NANO $3.53 \mu \mathrm{m}$ 


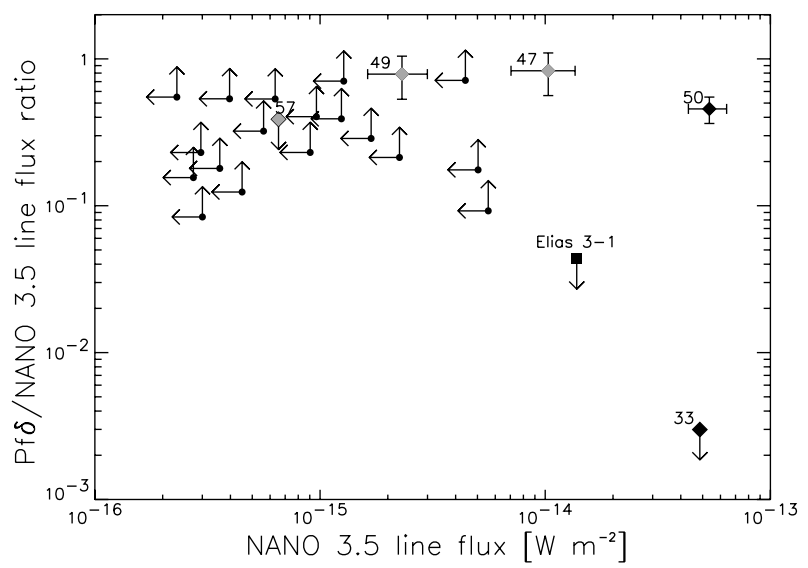

Fig. 7. The line flux ratio of $\operatorname{Pf} \delta$ and NANO $3.53 \mu \mathrm{m}$ versus the NANO $3.53 \mu \mathrm{m}$ line flux. Legend see Fig. 6.

line flux. Group I members HD 97048 and Elias 3-1 have no detected $\operatorname{Pf} \delta$ line. The massive young stellar object MWC 297 does display both features. We have indicated the measurements for the tentative nanodiamond emitters (V921 Sco, HD 163296 and $\mathrm{T} \mathrm{CrA}$ ) with grey plotting symbols. Also for these three targets, there is no uniformity. The early-type embedded source V921 Sco and group II member HD 163296 display the $\operatorname{Pf} \delta$ line, while the UXOR object T CrA does not.

$\mathrm{X}$-ray emission in stars with convective envelopes, like the lower-mass T Tauri stars, is often explained as being due to the presence of a magnetic field. The matter is less clear for the earlier-type Herbig stars. Although these stars are believed to have radiative envelopes and thus cannot drive a solar-like magnetic dynamo, the X-ray luminosity of Herbig stars is nevertheless explained in the light of magnetic effects. Such a magnetic field can be fossile (e.g. Skinner et al. 2004; Hamaguchi et al. 2005), or produced differently than in our Sun (e.g. the differential rotation dynamo model, Tout \& Pringle 1995). Hard and soft X-ray spectra are observed in the group of HAEBE stars. Generally, the first kind is attributed to hot plasma in a stellar corona or the presence of a late-type companion, the second to accretion events onto the photosphere. In O-type stars, energetic wind shocks can produce X-ray emission. It appears that Herbig winds are not powerful enough to produce the same effect (Skinner et al. 2004; Hamaguchi et al. 2005).

Whatever mechanism causes the X-ray luminosity in Herbig stars, it does not seem to influence the presence/absence of the nanodiamond features. In Fig. 8, we have plotted the X-ray luminosity versus the NANO 3.5 line luminosity. The X-ray luminosities are taken from Skinner et al. (2004), Hamaguchi et al. (2005) and Damiani et al. (2006). It appears that, in this small sample of observed Herbig stars, the (candidate) nanodiamond emitters have typical X-ray luminosities. When rescaling the NANO 3.5 line flux to the appropriate distance to the source, MWC 297 appears to be the most luminous nanodiamond target $\left(L_{3.5}=0.10 L_{\odot}\right)$. It is a rather strong X-ray source, although the X-rays likely emanate from nearby lower-mass stars (we follow the suggestion that $5 \%$ or less of the total X-ray luminosity around MWC 297 emanates from the B1.5 star itself, Vink et al. 2005; Damiani et al. 2006). Elias 3-1 has a comparable $\mathrm{X}$-ray luminosity, but a $3.53 \mu \mathrm{m}$ luminosity which is ten times smaller. HD 97048, the third confirmed nanodiamond emitter, has an intermediate nanodiamond luminosity $\left(L_{3.5}=0.05 L_{\odot}\right)$, but an X-ray luminosity 15 times more modest than the previous two targets. Recently, Elias 3-1 has been identified as a binary

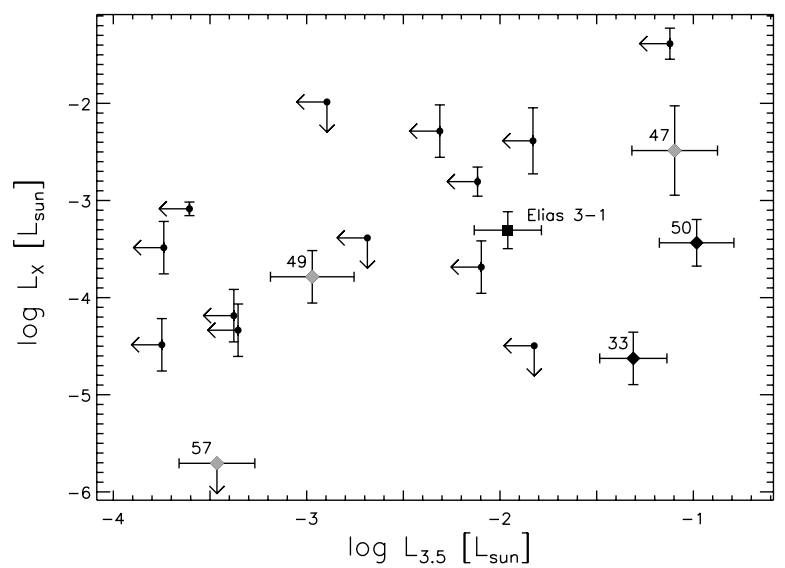

Fig. 8. The X-ray luminosity $L_{X}$ versus the NANO $3.53 \mu \mathrm{m}$ line luminosity $L_{3.5}$. The latter quantity is the line flux rescaled by a factor $4 \pi d^{2}$, with $d$ the distance to the source as listed in Skinner et al. (2004), Hamaguchi et al. (2005) or Damiani et al. (2006). We apply the same distance factor to the $3.53 \mu \mathrm{m}$ line flux as was applied for the cited $L_{\mathrm{X}}$, to avoid systematic errors. The plotting symbols are the same as in Fig. 6. The (candidate) nanodiamond emitting sources have X-ray luminosities which are not uncommon in the group of sample stars observed in the X-ray wavelength range.

(with a separation of $9 \mathrm{AU}$ at a distance of $160 \mathrm{pc}$, Smith et al. 2005). The companion is a lower-mass star, possibly with a convective envelope. The latter may explain the rather high X-ray luminosity attributed to Elias 3-1.

The analysis presented in the previous sections suggests that the few sources which display nanodiamond features are not exceptional compared to the bulk of Herbig stars. Furthermore, these targets do not appear to have obvious stellar or circumstellar characteristics in common.

\section{Conclusions and discussion}

We summarize the two main conclusions of the present paper.

- Our survey of 3 micron spectra of HAEBE stars has not revealed a new spectacular nanodiamond source like HD 97048 or Elias 3-1. This negative result increases the rarity of these near-IR bands in the group of Herbig stars. Within the current sample, only $4 \%$ of the targets are surrounded by detected nanodiamonds. We find a large range in nanodiamond luminosity, which spans 1-2 orders of magnitude. No clear correlation is found between the presence of the nanodiamond features and the disk and stellar parameters, although a weak link between nanodiamond luminosity and stellar luminosity may be present. Furthermore, there does not appear to be a specific disk/star property which the nanodiamond sources have in common. We have shown that our findings are inconclusive with respect to the influence of the surroundings of the targets. We do not find any evidence for a correlation of nanodiamond emitters with the locations of known recent supernova remnants, nor do we find evidence for an enrichment in heavy elements of the photosphere of the system with the most prominent nano-diamond features, HD 97048.

- Most to all Herbig stars with a flared disk are PAH emitters. This conclusion once again confirms the suggestion of Meeus et al. (2001) that PAH molecules can only be excited in systems where they can capture direct stellar UV photons. In the case of self-shadowed disks, the puffed-up 
Table 5. The detected features in the 3 micron region of the sample stars. The sample stars have been classified in Meeus groups I and II (Meeus et al. 2001). Targets with $A_{V}$ exceeding 4 are classified as embedded sources (E). Sources with unknown or uncertain classification are flagged with "?". The measured line fluxes and peak-over-continuum ratios of four spectral features are given: the Pfund $\delta$ emission line at $3.296 \mu \mathrm{m}$, the PAH $3.3 \mu \mathrm{m}$ feature and the nanodiamond features at 3.43 and $3.53 \mu \mathrm{m}$. Tentative detections (line flux between $1-3 \sigma$ ) are indicated with $a$. The flag $b$ indicates detected features of unclear origin, either linked to data reduction artefacts, nanodiamonds or an unknown carrier.

\begin{tabular}{|c|c|c|c|c|c|c|c|c|c|c|}
\hline$\overline{\# \#}$ & Target & Group & $\begin{array}{c}\operatorname{Pf} \delta \\
3.296 \mu \mathrm{m}\end{array}$ & $\begin{array}{c}\text { PAH } \\
3.3 \mu \mathrm{m} \\
\text { line flux } / 10\end{array}$ & $\begin{array}{c}\text { NANO } \\
3.43 \mu \mathrm{m} \\
16 \mathrm{~W} \mathrm{~m}^{-2}\end{array}$ & $\begin{array}{l}\text { NANO } \\
3.53 \mu \mathrm{m}\end{array}$ & $\begin{array}{c}\operatorname{Pf} \delta \\
3.296 \mu \mathrm{m}\end{array}$ & $\begin{array}{c}\text { PAH } \\
3.3 \mu \mathrm{m} \\
\text { peak-over-con }\end{array}$ & $\begin{array}{c}\text { NANO } \\
3.43 \mu \mathrm{m} \\
\text { nuum ratio }\end{array}$ & $\begin{array}{l}\text { NANO } \\
3.53 \mu \mathrm{m}\end{array}$ \\
\hline 1 & UX Ori & II & $0.7 \pm 0.2$ & $<14$ & $<16$ & $<2.9$ & $1.18 \pm 0.05$ & $<1.05$ & $<1.05$ & $<1.01$ \\
\hline 2 & HD 34282 & I & $<1.1$ & $24 \pm 7$ & $<17$ & $<4.7$ & $<1.06$ & $1.22 \pm 0.07$ & $<1.06$ & $<1.02$ \\
\hline 3 & V346 Ori & I & $<1.4$ & $17 \pm 8^{a}$ & $<23$ & $<8.4$ & $<1.10$ & $1.2 \pm 0.1$ & $<1.10$ & $<1.05$ \\
\hline 4 & CO Ori & II & $<0.4$ & $<6.9$ & $<8.2$ & $1.2 \pm 0.7^{a, b}$ & $<1.05$ & $<1.05$ & $<1.15$ & $1.02 \pm 0.02$ \\
\hline 5 & HD 35929 & II & $0.4 \pm 0.4^{a}$ & $<12$ & $<14$ & $<2.7$ & $1.04 \pm 0.04$ & $<1.04$ & $<1.04$ & $<1.01$ \\
\hline 6 & $\mathrm{StH} \alpha 41$ & II? & $<0.32$ & $<5.8$ & $<10$ & $<4.2$ & $<1.05$ & $<1.05$ & $<1.15$ & $<1.05$ \\
\hline 7 & HK Ori & II & $<1.1$ & $<31$ & $<35$ & $<9.0$ & $<1.08$ & $<1.08$ & $<1.08$ & $<1.03$ \\
\hline 8 & HD 244604 & II & $<1.5$ & $<27$ & $<31$ & $<8.0$ & $<1.10$ & $<1.10$ & $<1.10$ & $<1.04$ \\
\hline 9 & RY Ori & II & $<0.25$ & $<4.8$ & $<5.7$ & $<1.1$ & $<1.06$ & $<1.06$ & $<1.06$ & $<1.02$ \\
\hline 10 & $\mathrm{~T}$ Ori & II? & $<3.7$ & $<62$ & $<69$ & $<12$ & $<1.06$ & $<1.06$ & $<1.06$ & $<1.01$ \\
\hline 11 & V380 Ori & II? & $5 \pm 1$ & $<86$ & $<110$ & $<22$ & $1.27 \pm 0.08$ & $<1.07$ & $<1.08$ & $<1.02$ \\
\hline 12 & V586 Ori & II & $0.25 \pm 0.09^{a}$ & $<8.7$ & $<10$ & $<3.0$ & $1.13 \pm 0.05$ & $<1.05$ & $<1.05$ & $<1.02$ \\
\hline 13 & BF Ori & II & $0.6 \pm 0.2$ & $<7.6$ & $<8.8$ & $<3.5$ & $1.15 \pm 0.05$ & $<1.05$ & $<1.05$ & $<1.03$ \\
\hline 14 & HD 37357 & II & $2.1 \pm 0.4$ & $<6.2$ & $<7.1$ & $<3.9$ & $1.15 \pm 0.03$ & $<1.03$ & $<1.03$ & $<1.02$ \\
\hline 15 & N3sk 81 & $\mathrm{E}$ & $<0.58$ & $<9.7$ & $<11$ & $<3.1$ & $<1.04$ & $<1.04$ & $<1.04$ & $<1.02$ \\
\hline 16 & HD 37411 & II & $<0.65$ & $<10$ & $<12$ & $<4.4$ & $<1.04$ & $<1.04$ & $<1.04$ & $<1.02$ \\
\hline 17 & Haro $13 a$ & I & $<1.9$ & $<34$ & $<44$ & $<7.3$ & $<1.03$ & $<1.03$ & $<1.03$ & $<1.01$ \\
\hline 18 & V599 Ori & E & $<0.24$ & $5 \pm 1$ & $<4.2$ & $<0.95$ & $<1.04$ & $1.15 \pm 0.04$ & $<1.04$ & $<1.01$ \\
\hline 19 & V350 Ori & II & $<0.063$ & $<1.1$ & $<1.2$ & $<0.37$ & $<1.06$ & $<1.06$ & $<1.06$ & $<1.02$ \\
\hline 20 & HD 37806 & II & $1.3 \pm 0.3$ & $<17$ & $<20$ & $<2.3$ & $1.14 \pm 0.03$ & $<1.03$ & $<1.03$ & $<1.01$ \\
\hline 21 & HD 250550 & I & $<3.3$ & $<59$ & $<69$ & $<8.3$ & $<1.16$ & $<1.16$ & $<1.16$ & $<1.03$ \\
\hline 22 & VY Mon & E & $<31$ & $<530$ & $<670$ & $<38$ & $<1.17$ & $<1.17$ & $<1.17$ & $<1.01$ \\
\hline 23 & $\mathrm{LkH} \alpha 215$ & I & $<0.40$ & $<7.3$ & $<8.8$ & $<1.7$ & $<1.07$ & $<1.07$ & $<1.07$ & $<1.02$ \\
\hline 24 & HD 259431 & I & $0.6 \pm 0.2^{a}$ & $<32$ & $<37$ & $<4.5$ & $1.16 \pm 0.06$ & $<1.06$ & $<1.06$ & $<1.01$ \\
\hline 25 & R Mon & I & $<13$ & $<260$ & $<350$ & $28 \pm 10^{a, b}$ & $<1.08$ & $<1.08$ & $<1.08$ & $1.03 \pm 0.01$ \\
\hline 26 & V590 Mon & ? & $<1.9$ & $7 \pm 6^{a}$ & $<34$ & $2.4 \pm 0.8^{a, b}$ & $<1.16$ & $1.2 \pm 0.2$ & $<1.16$ & $1.05 \pm 0.02$ \\
\hline 27 & GU CMa & II & $4.9 \pm 0.8$ & $<43$ & $<48$ & $<12$ & $1.46 \pm 0.09$ & $<1.08$ & $<1.08$ & $<1.03$ \\
\hline 28 & HD 53367 & ? & $<1.3$ & $<20$ & $<24$ & $<5.1$ & $<1.11$ & $<1.11$ & $<1.11$ & $<1.03$ \\
\hline 29 & NX Pup & II & $<3.4$ & $<67$ & $<75$ & $<15$ & $<1.04$ & $<1.04$ & $<1.04$ & $<1.01$ \\
\hline 30 & HD 76534 & ? & $<0.27$ & $<4.5$ & $<5.2$ & $<0.59$ & $<1.19$ & $<1.19$ & $<1.19$ & $<1.03$ \\
\hline 31 & HD 85567 & II & $<1.2$ & $<19$ & $<21$ & $<3.0$ & $<1.06$ & $<1.06$ & $<1.06$ & $<1.01$ \\
\hline 32 & HD 95881 & II & $<3.1$ & $<49$ & $<56$ & $<9.9$ & $<1.10$ & $<1.10$ & $<1.10$ & $<1.02$ \\
\hline 33 & HD 97048 & I & $<1.5$ & $65 \pm 20$ & $200 \pm 20$ & $486 \pm 5$ & $<1.10$ & $1.4 \pm 0.1$ & $2.0 \pm 0.1$ & $4.3 \pm 0.2$ \\
\hline 34 & HD 100546 & I & $9 \pm 2$ & $240 \pm 40$ & $<94$ & $13 \pm 10^{a, b}$ & $1.33 \pm 0.06$ & $1.34 \pm 0.06$ & $<1.06$ & $1.05 \pm 0.04$ \\
\hline 35 & HD 101412 & II & $<1.3$ & $13 \pm 10^{a}$ & $<25$ & $<7.5$ & $<1.06$ & $1.08 \pm 0.06$ & $<1.06$ & $<1.03$ \\
\hline 36 & T Cha & II & $<0.85$ & $<16$ & $<17$ & $<4.0$ & $<1.07$ & $<1.07$ & $<1.07$ & $<1.02$ \\
\hline 37 & LSS 3027 B & ? & $<0.46$ & $<8.7$ & $<8.8$ & $<3.6$ & $<1.73$ & $<1.73$ & $<1.73$ & $<1.39$ \\
\hline 38 & HD 130437 & $?$ & $1.8 \pm 0.2$ & $<6.5$ & $<7.1$ & $<5.6$ & $1.64 \pm 0.06$ & $<1.06$ & $<1.06$ & $<1.06$ \\
\hline 39 & SS73 44 & I & $<0.89$ & $<16$ & $<17$ & $<8.8$ & $<1.05$ & $<1.05$ & $<1.05$ & $<1.04$ \\
\hline 40 & HD 132947 & II & $<0.68$ & $<8.6$ & $<9.1$ & $<8.1$ & $<1.14$ & $<1.14$ & $<1.14$ & $<1.18$ \\
\hline 41 & HD 141569 & II & $<0.87$ & $<37$ & $<38$ & $<5.9$ & $<1.20$ & $<1.20$ & $<1.20$ & $<1.05$ \\
\hline 42 & HD 142666 & II & $<0.72$ & $<20$ & $<21$ & $<5.8$ & $<1.04$ & $<1.04$ & $<1.04$ & $<1.02$ \\
\hline 43 & HD 142527 & I & $32 \pm 5$ & $<110$ & $<120$ & $44 \pm 9^{b}$ & $1.27 \pm 0.04$ & $<1.04$ & $<1.04$ & $1.05 \pm 0.01$ \\
\hline 44 & HR 5999 & II & $5 \pm 3^{a}$ & $<110$ & $<150$ & $<55$ & $1.08 \pm 0.04$ & $<1.04$ & $<1.04$ & $<1.02$ \\
\hline 45 & HD 150193 & II & $3.4 \pm 0.3$ & $<13$ & $<15$ & $<6.3$ & $1.15 \pm 0.01$ & $<1.01$ & $<1.01$ & $<1.01$ \\
\hline 46 & AK Sco & II & $<0.79$ & $<13$ & $<15$ & $2.5 \pm 0.9^{a, b}$ & $<1.03$ & $<1.03$ & $<1.03$ & $1.03 \pm 0.01$ \\
\hline 47 & V921 Sco & E & $86 \pm 5$ & $<160$ & $<190$ & $103 \pm 30^{b}$ & $1.42 \pm 0.03$ & $<1.03$ & $<1.08$ & $1.04 \pm 0.01$ \\
\hline 48 & KK Oph & II & $<2.8$ & $<47$ & $<66$ & $<19$ & $<1.05$ & $<1.05$ & $<1.05$ & $<1.02$ \\
\hline 49 & HD 163296 & II & $18 \pm 3$ & $<120$ & $<130$ & $23 \pm 7^{b}$ & $1.16 \pm 0.03$ & $<1.03$ & $<1.03$ & $1.03 \pm 0.01$ \\
\hline 50 & MWC 297 & E & $240 \pm 20$ & $<910$ & $<1100$ & $535 \pm 100$ & $1.63 \pm 0.05$ & $<1.04$ & $<1.04$ & $1.06 \pm 0.01$ \\
\hline 51 & VV Ser & II & $2.1 \pm 0.4$ & $<23$ & $<25$ & $<9.0$ & $1.13 \pm 0.03$ & $<1.03$ & $<1.03$ & $<1.01$ \\
\hline 52 & MWC 300 & E & $9 \pm 1$ & $<82$ & $<97$ & $50 \pm 9^{b}$ & $1.13 \pm 0.02$ & $<1.02$ & $<1.02$ & $1.03 \pm 0.01$ \\
\hline 53 & AS 310 & I & $<0.83$ & $11 \pm 3$ & $<9.5$ & $<4.0$ & $<1.34$ & $2.1 \pm 0.5$ & $<1.34$ & $<1.22$ \\
\hline 54 & $\mathrm{~S} \mathrm{CrA}$ & $?$ & $3.9 \pm 0.4$ & $<32$ & $<35$ & $10 \pm 3^{b}$ & $1.20 \pm 0.03$ & $<1.03$ & $<1.03$ & $1.03 \pm 0.01$ \\
\hline 55 & TY CrA & $?$ & $<0.75$ & $40 \pm 4$ & $<12$ & $<4.7$ & $<1.03$ & $1.28 \pm 0.03$ & $<1.03$ & $<1.02$ \\
\hline 56 & $\mathrm{R} \mathrm{CrA}$ & I & $<26$ & $<470$ & $<550$ & $290 \pm 50^{b}$ & $<1.02$ & $<1.02$ & $<1.02$ & $1.04 \pm 0.01$ \\
\hline 57 & $\mathrm{~T} \mathrm{CrA}$ & II? & $<2.5$ & $<44$ & $<96$ & $7 \pm 1^{b}$ & $<1.06$ & $<1.06$ & $<1.19$ & $1.04 \pm 0.01$ \\
\hline 58 & HD 179218 & I & $<3.1$ & $160 \pm 20$ & $<47$ & $<6$ & $<1.03$ & $1.28 \pm 0.03$ & $<1.03$ & $<1.04$ \\
\hline 59 & НВС 687 & I? & $<0.14$ & $1.7 \pm 0.7^{a}$ & $<2.8$ & $<1.0$ & $<1.07$ & $1.14 \pm 0.07$ & $<1.07$ & $<1.03$ \\
\hline 60 & V1295 Aql & II & $4.8 \pm 0.6$ & $<40$ & $<44$ & $<16$ & $1.18 \pm 0.02$ & $<1.02$ & $<1.02$ & $<1.01$ \\
\hline
\end{tabular}


inner rim casts a shadow over the outer disk, preventing the PAH molecules from being heated by the central star. Our findings reinforce the results of AV04 and Habart et al. (2004a). The latter studies were based on ISO data. Due to the large aperture sizes of the ISO instruments, it is difficult to differentiate between PAH emission emanating from the disk surface, and contamination from the wider surroundings of the target. The present ISAAC data set, which clearly confirms the correlation, is considerably more reliable in this sense: both the narrow slit and the chop-nod technique, which allows for an efficient subtraction of constant background emission, reduce the possibility of confusion with extended PAH emission drastically.

Laboratory experiments have been instrumental in the identification of the carriers of the 3 micron features (e.g. Guillois et al. 1999; Sheu et al. 2002; Jones et al. 2004; Mutschke et al. 2004). Currently, the astronomical spectra can be reproduced from the absorption spectra of films of hydrogen-terminated diamonds (Sheu et al. 2002). The spectral features in the 3 micron range are due to $\mathrm{CH}$-bond stretches (as is the PAH $3.3 \mu \mathrm{m}$ feature). The prominent peaks around 3.43 and $3.53 \mu$ m observed in HAEBE spectra can only be reproduced when the diamonds are sufficiently large. Typical sizes for the diamonds in HAEBE stars $(\sim 50-100 \mathrm{~nm})$ are 2 orders of magnitude larger than the nanodiamonds found in our solar system (e.g. Jones et al. 2004). The spectral features hence indicate the presence of large, hot hydrogen-terminated diamonds.

The question remains whether there are indeed no diamonds in the majority of disks. It may be possible that nanodiamonds of small sizes are present, but remain undetected, because their spectral signature is less pronounced (e.g. Sheu et al. 2002). Also diamonds which are not hydrogen-terminated are not detectable in the 3 micron region. Diamonds that are stripped from hydrogen atoms are hence overlooked by our survey. A third possibility why (nano)diamonds can remain undetected is their exact location - and hence temperature - in the disk. Cool hydrogen-terminated diamonds will not radiate significantly in the near-IR. The absence of the 3 micron features in the spectrum of a HAEBE system hence does not necessarily mean that no (nano)diamonds are present.

\section{In-situ formation or extraneous origin?}

Diamond was the first presolar mineral to be identified in meteorites (Lewis et al. 1987) and it has the highest relative abundance among carbonaceous grains. The diamond has been classified as presolar based on the presence of a significant overabundance of very heavy $(\mathrm{Xe}-\mathrm{H})$ and very light $(\mathrm{Xe}-\mathrm{L})$ $\mathrm{Xe}$ isotopes (together Xe-HL). The overabundance of this noblegas carrier is expected to be produced by $r$ - and $p$-process nucleosynthesis in supernovae. The link between the presence of nanodiamonds and the xenon overabundance led to the hypothesis that the diamonds are formed in, and injected into the system by a supernova outflow (Clayton 1989). Isotope anomalies on other heavy elements such as Te and Pd also point to supernova nucleosynthesis (Richter et al. 1998; Maas et al. 2001).

Recent analysis of ${ }^{60} \mathrm{Fe}$ isotopes in chondrites has clearly demonstrated that a nearby type II supernova has contributed material to the natal cloud from which our own solar system formed (Mostefaoui et al. 2005; Tachibana et al. 2006). This process may have been important for planet formation, as the the radioactive decay of ${ }^{60} \mathrm{Fe}$ into ${ }^{60} \mathrm{Ni}$ was an important heat source for the early planetary melting and differentiation and keeping asteroids thermally active for much longer than would be possible from the decay of ${ }^{26} \mathrm{Al}$ alone.

Infrared spectroscopy has shown that solar-system diamonds contain $\mathrm{N}$ and $\mathrm{O}$, probably in chemical functional groups on their surfaces (Lewis et al. 1989; Mutschke et al. 1995; Andersen et al. 1998; Jones et al. 2004). However, the similarity of C- and $\mathrm{N}$-isotopes of these diamonds and the solar system as a whole supports the idea that not all solar-system diamonds originate from supernovae and that the supernova contribution to the diamonds in our own solar system is probably not very large. It thus remains unclear how the majority of the solar-system diamonds has formed.

Although advocated by several recent studies (Kouchi et al. 2005; Binette et al. 2005), the presence of nanodiamonds in the interstellar medium remains controversial. The absence of observable 3.43 and $3.53 \mu \mathrm{m}$ features in the ISM suggests that hydrogenated hydrocarbons cannot be more abundant than $\approx 0.1 \mathrm{ppm}$ in the interstellar medium (Tielens et al. 1987). Van Kerckhoven et al. (2002) showed that in HD 97048 and Elias 3-1, 1 nanodiamond part per billion relative to hydrogen is required to reproduce the 3 micron spectra observed by ISO, making an ISM origin of nanodiamonds in principle a viable option. This hypothesis has problems in explaining the paucity of detections of the 3.43 and $3.53 \mu \mathrm{m}$ feature in Herbig stars within our sample, however.

We found no evidence for the presence of a supernova remnant (SNR) in the proximity of any of our sample sources. We made use of the catalog of galactic SNRs published by Green (2004). Only R Mon and V590 Mon are potentially located close to a SNR; the Monoceros Nebula (G205.5+0.5). These sources display no detected nanodiamond features, however. We note that the absence of a SNR in the vicinity of our sample stars in the above-mentioned catalog does not necessarily imply that no supernova has actually occured. Most of the sample targets are located in massive-star-forming regions. It may very well be that the most massive members in such a region have recently gone off as a supernova. In a relatively crowded star forming region, the ejecta of such an event likely affect a number of surrounding young stellar objects or molecular clouds. In this scenario, the presence of diamonds in the circumstellar disk is expected to be common to all disk systems in the vicinity of the supernova. Unfortunately, we have no spectra of disk sources close to Elias 3-1, HD 97048 or MWC 297 to check this hypothesis.

The parent star of a disk which contains supernova-injected diamonds must be be polluted by supernova material as well, under the supernova hypothesis. Enrichment of the stellar photosphere by a supernova outflow is expected to alter the photospheric abundance pattern of the star significantly. An abundance analysis of the strongest diamond source HD 97048 has produced upper limits on the abundances of a few heavy elements. The Sr abundance is at least one order of magnitude less than the solar abundance of this element. No evidence supporting the supernova hypothesis is found.

Except for HD 97048 and Elias 3-1, both nanodiamond features have also been observed in the post-AGB binary HR 4049 (Geballe et al. 1989). The post-AGB phase is very short $(\sim 10000 \mathrm{yr})$ compared to the lifetime of an intermediate-mass pre-main-sequence star. Due to the short timescales, it is unlikely that a nearby supernova is the cause of the presence of nanodiamonds in this system. However, the oxygen isotopes in the circumbinary disk of HR 4049 display peculiar behaviour: the relative ${ }^{17} \mathrm{O}$ and ${ }^{18} \mathrm{O}$ abundances are two orders of magnitude larger than the surface abundances in other evolved stars $\left({ }^{16} \mathrm{O} /{ }^{17} \mathrm{O}=\right.$ $8.3 \pm 2.3$ and ${ }^{16} \mathrm{O} /{ }^{18} \mathrm{O}=6.9 \pm 0.9$, Cami \& Yamamura 2001). 
These exceptional values are most likely related to the binary nature of HR 4049, and possibly to a nova outburst. The presence of nanodiamonds in the circumstellar environment may be related to such an event as well.

The extraneous origin of the circumstellar diamonds is disputed, however. Dai et al. (2002) have stated that, if the supernova hypothesis is true, nanodiamonds should be abundant in solar-system comets as well. These objects are believed to have formed further out in the early solar system and are likely more pristine than meteorites. The authors have investigated fragile, carbon-rich IPD particles which enter the Earth's atmosphere with speeds in the range of cometary bodies, which suggests that the grains originate from these objects. Nanodiamonds are absent or strongly depleted in such IPD grains, which indicates that diamonds are not uniformly abundant in the solar system. This may support the hypothesis that the detected meteoritic nanodiamonds have formed in situ, and are not of presolar origin.

Goto et al. (2000) has suggested that the X-ray hardness of the radiation field of the central star may play a decisive role in the formation of diamond. However, our analysis has shown no obvious link between the source's X-ray luminosity and presence/absence of nanodiamonds. Furthermore, the strength of the few detected diamond features appears to be independent from the X-ray strength of the central star as well.

The extraneous origin of nanodiamonds is an attractive hypothesis, because it naturally explains the paucity of nanodiamond sources and the seemingly very different properties of these targets. No evidence was found, however, to substantiate the claim of an external triggering source in the vicinity of the targets. In-situ formation of nanodiamonds is a viable alternative. However, it remains unclear why only very few disks can/could provide the specific conditions needed to produce diamond. The disk and stellar parameters of the diamond sources are not uncommon, nor do the targets form a consistent group within the Herbig stars. Selection effects in terms of the size, temperature or hydrogenation of the diamonds may nonetheless be present. Such effects could prevent the detection of features in the 3 micron region in spectra of the majority of circumstellar disks. A larger sample of systems displaying diamond emission is needed to distinguish between in-situ formation and an extraneous origin of the (nano)diamonds in Herbig Ae/Be stars and in our own solar system.

Acknowledgements. The authors thank R. van Boekel for kindly providing us with the TIMMI2 spectrum of V921 Sco. Atomic data compiled in the DREAM data base (Biémont et al. 1999) were extracted via VALD (Kupka et al. 1999, and references therein).

\section{References}

Acke, B., \& van den Ancker, M. E. 2004, A\&A, 426, 151

Acke, B., \& Waelkens, C. 2004, A\&A, 427, 1009

Acke, B., \& van den Ancker, M. E. 2006, A\&A, 449, 267

Acke, B., van den Ancker, M. E., Dullemond, C. P., van Boekel, R., \& Waters, L. B. F. M. 2004, A\&A, 422, 621

Andersen, A. C., Jorgensen, U. G., Nicolaisen, F. M., Sorensen, P. G., \& Glejbol, K. 1998, A\&A, 330, 1080

Biémont, E., Palmeri, P., \& Quinet, P. 1999, Ap\&SS, 269, 635

Bik, A., \& Thi, W. F. 2004, A\&A, 427, L13

Binette, L., Magris C., G., Krongold, Y., et al. 2005, ApJ, 631, 661

Cami, J., \& Yamamura, I. 2001, A\&A, 367, L1

Clayton, D. D. 1989, ApJ, 340, 613

Dai, Z. R., Bradley, J. P., Joswiak, D. J., et al. 2002, Nature, 418, 157

Damiani, F., Micela, G., \& Sciortino, S. 2006, A\&A, 447, 1041

Dullemond, C. P. 2002, A\&A, 395, 853

Dullemond, C. P., van den Ancker, M. E., Acke, B., \& van Boekel, R. 2003, ApJ, 594, L47
Finkenzeller, U., \& Mundt, R. 1984, A\&AS, 55, 109

Fuente, A., Neri, R., Martín-Pintado, J., et al. 2001, A\&A, 366, 873

Fuente, A., Rodríguez-Franco, A., Testi, L., et al. 2003, ApJ, 598, L39

Geballe, T. R., Noll, K. S., Whittet, D. C. B., \& Waters, L. B. F. M. 1989, ApJ, 340, L29

Goto, M., Maihara, T., Terada, H., et al. 2000, A\&AS, 141, 149

Green, D. A. 2004, Bulletin of the Astronomical Society of India, 32, 335

Grinin, V. P., Kiselev, N. N., Minikulov, N. H., \& Chernova, G. P. 1988, Pis ma Astronomicheskii Zhurnal, 14, 514

Grinin, V. P., Rostopchina, A. N., \& Shakhovskoi, D. N. 1998, Astron. Lett., 24, 802

Guillois, O., Ledoux, G., \& Reynaud, C. 1999, ApJ, 521, L133

Habart, E., Natta, A., \& Krügel, E. 2004a, A\&A, 427, 179

Habart, E., Testi, L., Natta, A., \& Carbillet, M. 2004b, ApJ, 614, L129

Habart, E., Testi, L., Natta, A., \& Vanzi, L. 2003, A\&A, 400, 575

Hamaguchi, K., Yamauchi, S., \& Koyama, K. 2005, ApJ, 618, 360

Houk, N., \& Cowley, A. P. 1975, Michigan Catalogue of two-dimensional spectral types for the HD star (Ann Arbor: University of Michigan, Departement of Astronomy, 1975)

Jones, A. P., d'Hendecourt, L. B., Sheu, S.-Y., et al. 2004, A\&A, 416, 235

Kessler, M. F., Steinz, J. A., Anderegg, M. E., et al. 1996, A\&A, 315, L27

Kouchi, A., Nakano, H., Kimura, Y., \& Kaito, C. 2005, ApJ, 626, L129

Kupka, F., Piskunov, N., Ryabchikova, T. A., Stempels, H. C., \& Weiss, W. W. 1999, A\&AS, 138, 119

Lewis, R. S., Ming, T., Wacker, J. F., Anders, E., \& Steel, E. 1987, Nature, 326, 160

Lewis, R. S., Anders, E., \& Draine, B. T. 1989, Nature, 339, 117

Maas, R., Loss, R. D., Rosman, K. J. R., et al. 2001, Meteoritics and Planetary Science, 36, 849

Malfait, K., Bogaert, E., \& Waelkens, C. 1998a, A\&A, 331, 211

Malfait, K., Waelkens, C., Waters, L. B. F. M., et al. 1998b, A\&A, 332, L25

Malfait, K., Waelkens, C., Bouwman, J., de Koter, A., \& Waters, L. B. F. M. 1999, A\&A, 345, 181

Mannings, V., \& Sargent, A. I. 1997, ApJ, 490, 792

Meeus, G., Waters, L. B. F. M., Bouwman, J., et al. 2001, A\&A, 365, 476

Mora, A., Merín, B., Solano, E., et al. 2001, A\&A, 378, 116

Mostefaoui, S., Lugmair, G. W., \& Hoppe, P. 2005, ApJ, 625, 271

Mutschke, H., Dorschner, J., Henning, T., Jager, C., \& Ott, U. 1995, ApJ, 454, L157

Mutschke, H., Andersen, A. C., Jäger, C., Henning, T., \& Braatz, A. 2004, A\&A, 423, 983

Natta, A., Testi, L., Neri, R., Shepherd, D. S., \& Wilner, D. J. 2004, A\&A, 416, 179

Piétu, V., Dutrey, A., \& Kahane, C. 2003, A\&A, 398, 565

Richter, S., Ott, U., \& Begemann, F. 1998, Nature, 391, 261

Sheu, S.-Y., Lee, I.-P., Lee, Y. T., \& Chang, H.-C. 2002, ApJ, 581, L55

Shore, S. N., Brown, D. N., Bopp, B. W., et al. 1990, ApJS, 73, 461

Siebenmorgen, R., Prusti, T., Natta, A., \& Müller, T. G. 2000, A\&A, 361, 258

Skinner, S. L., Güdel, M., Audard, M., \& Smith, K. 2004, ApJ, 614, 221

Smith, K. W., Balega, Y. Y., Duschl, W. J., et al. 2005, A\&A, 431, 307

Tachibana, S., Huss, G. R., Kita, N. T., Shimoda, G., \& Morishita, Y. 2006, ApJ, 639, L87

Terada, H., Imanishi, M., Goto, M., \& Maihara, T. 2001, A\&A, 377, 994

Testi, L., Natta, A., Shepherd, D. S., \& Wilner, D. J. 2003, A\&A, 403, 323

Thé, P. S., de Winter, D., \& Pérez, M. R. 1994, A\&AS, 104, 315

Tielens, A. G. G. M., Seab, C. G., Hollenbach, D. J., \& McKee, C. F. 1987, ApJ, 319, L109

Tout, C. A., \& Pringle, J. E. 1995, MNRAS, 272, 528

van Boekel, R., Waters, L. B. F. M., Dominik, C., et al. 2003, A\&A, 400, L21

van Boekel, R., Waters, L. B. F. M., Dominik, C., et al. 2004, A\&A, 418, 177

van den Ancker, M. E., de Winter, D., \& Tjin A Djie, H. R. E. 1998, A\&A, 330, 145

van den Ancker, M. E., Bouwman, J., Wesselius, P. R., et al. 2000a, A\&A, 357, 325

van den Ancker, M. E., Wesselius, P. R., \& Tielens, A. G. G. M. 2000b, A\&A, 355,194

Van Kerckhoven, C., Tielens, A. G. G. M., \& Waelkens, C. 2002, A\&A, 384, 568

Vink, J. S., Drew, J. E., Harries, T. J., \& Oudmaijer, R. D. 2002, MNRAS, 337, 356

Vink, J. S., O’Neill, P. M., Els, S. G., \& Drew, J. E. 2005, A\&A, 438, L21

Voshchinnikov, N. V. 1990, Astrophysics, 30, 309

Waelkens, C., Waters, L. B. F. M., de Graauw, M. S., et al. 1996, A\&A, 315, L245

Wenzel, W. 1968, In Non-Periodic Phenomena in Variable Stars, ed. L. Detre, IAU Coll., 4, 61 
B. Acke \& M. E. van den Ancker: Diamonds in Herbig Ae/Be systems, Online Material p 1

\section{Online Material}


B. Acke \& M. E. van den Ancker: Diamonds in Herbig Ae/Be systems, Online Material p 2
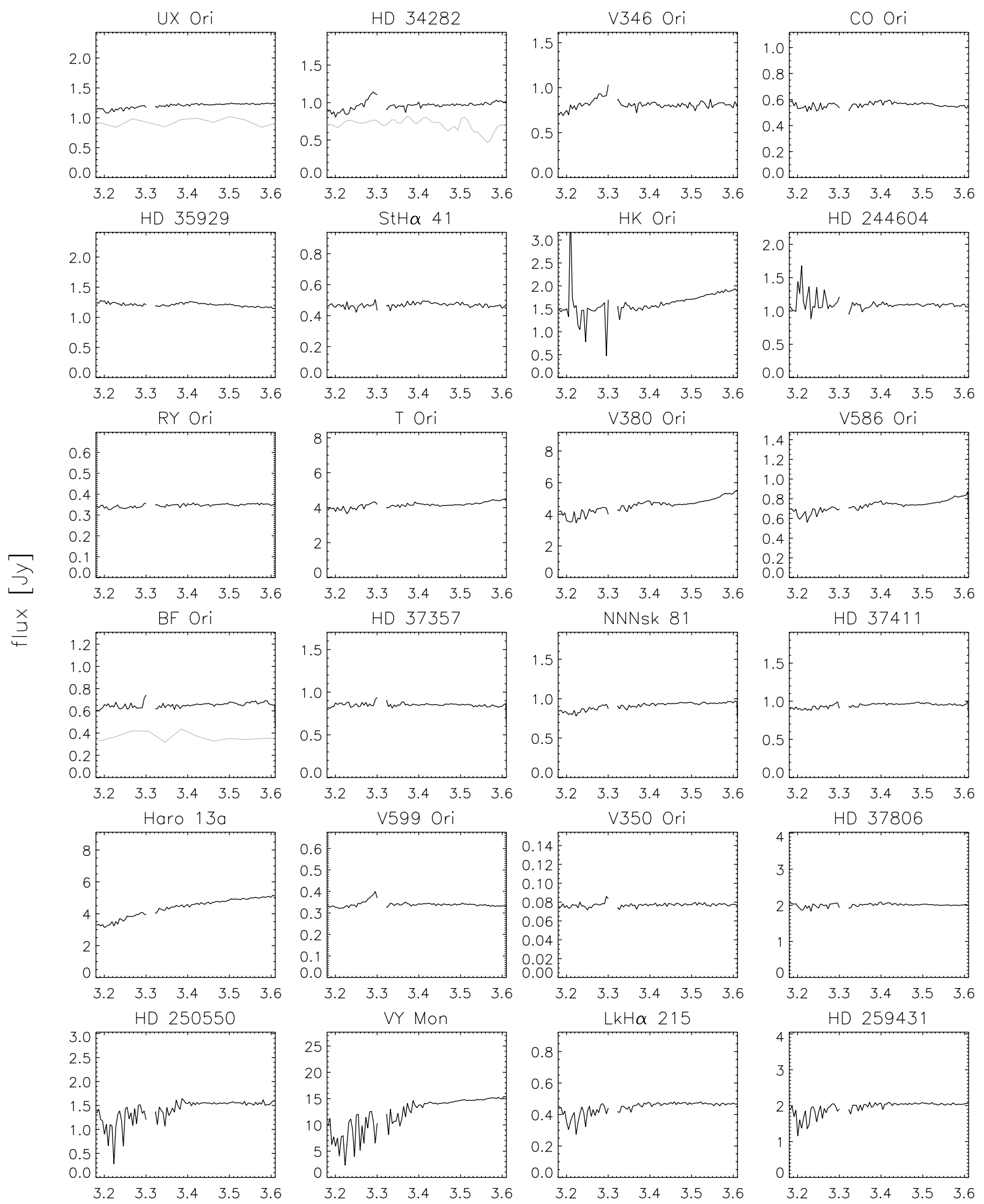

wavelength $[\mu \mathrm{m}]$

Fig. 1. The ISAAC spectra of the sample stars, rebinned to a resolution of 750. When available, ISO-SWS $(R=150)$ or PHT $-\mathrm{S}(R \sim 90)$ spectra are overplotted in grey. The part of the ISAAC spectrum between 3.3 and $3.32 \mu \mathrm{m}$ has been excluded. This part is unreliable due to the high atmospheric opacity in this wavelength range. 
B. Acke \& M. E. van den Ancker: Diamonds in Herbig Ae/Be systems, Online Material p 3
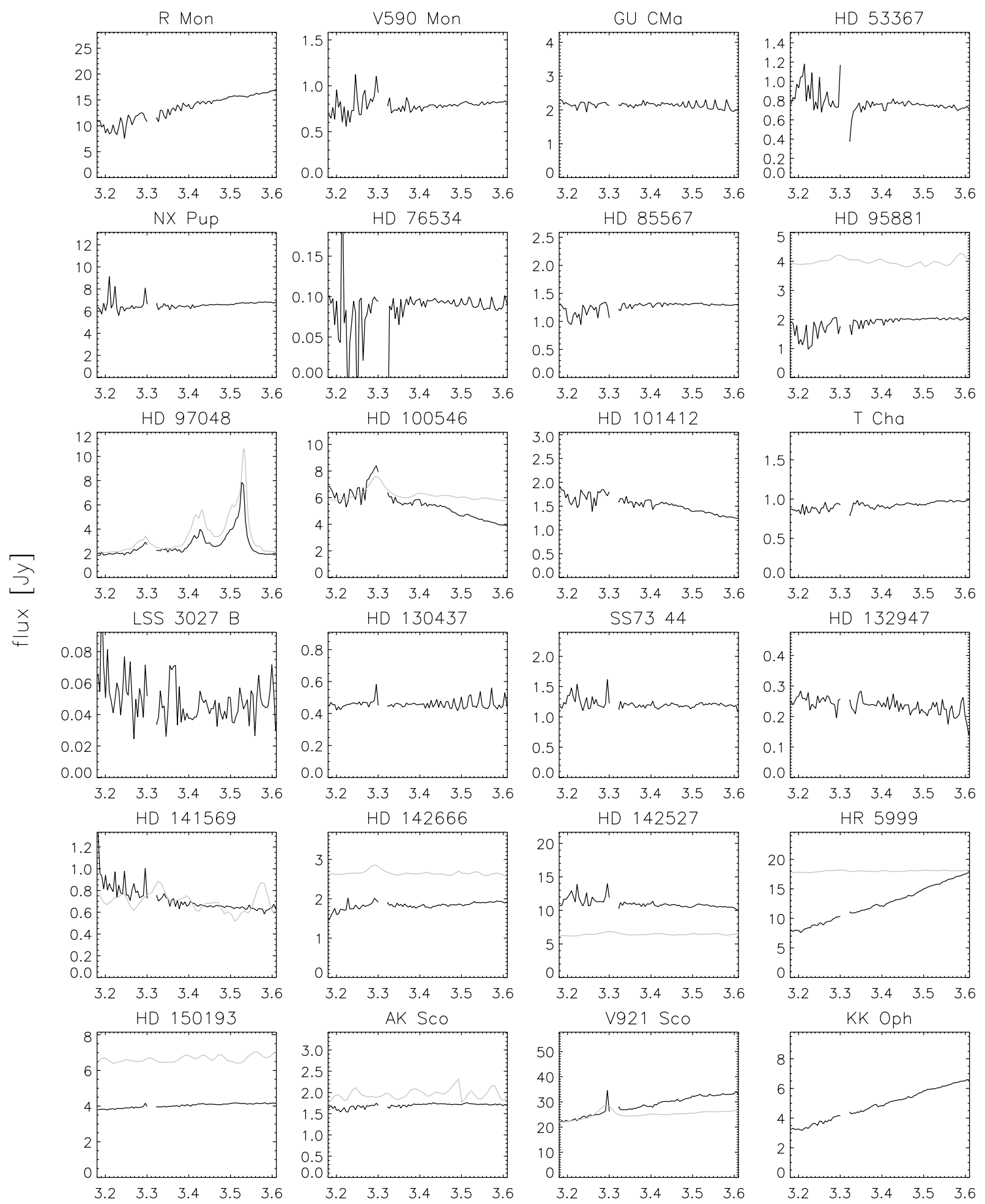

wavelength $[\mu \mathrm{m}]$

Fig. 1. continued. 
B. Acke \& M. E. van den Ancker: Diamonds in Herbig Ae/Be systems, Online Material p 4
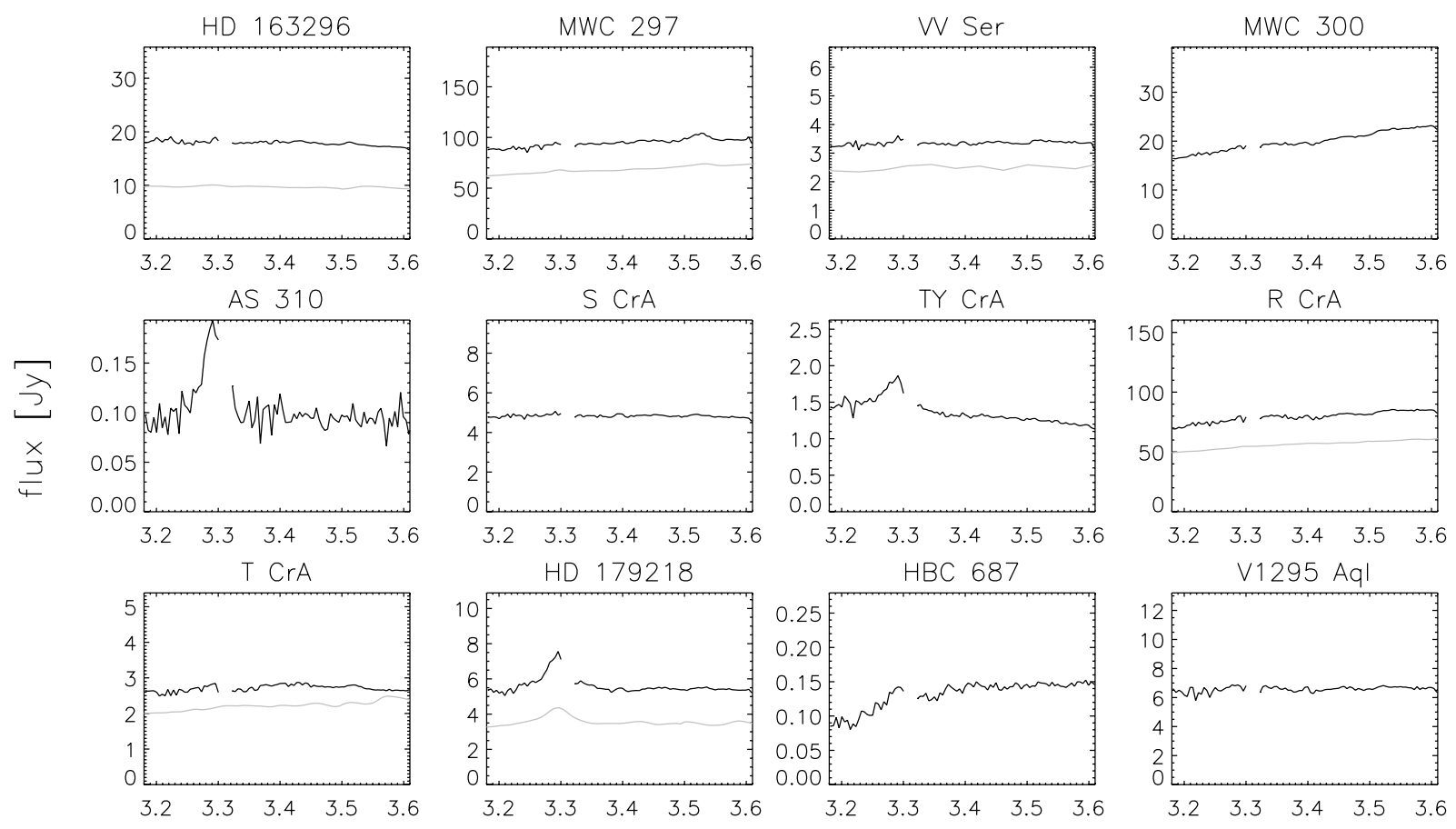

wavelength $[\mu \mathrm{m}]$

Fig. 1. continued. 
Table 4. The observation log of the sample stars The right ascension (RA), declination (Dec) and date of the ISAAC observations are indicated. The date, UT and integration time T of the observation are given. We have assigned a quality label (Q) to the ISAAC spectra: G-good quality; L-low flux levels $(<0.5 \mathrm{Jy})$; J-large flux-level jump ( $>50 \%)$ between the 3.3 and 3.5 micron spectrum. The seeing at $\lambda=0.5 \mu \mathrm{m}$, estimated by the VLT Astronomical Site Monitor (ASM), is given at the time of the 3.3 and $3.5 \mu \mathrm{m}$ ISAAC observations respectively. The final column lists the spectral calibrator stars. These standard stars have been observed during the same night as the science target, unless when flagged with $\star$.

\begin{tabular}{|c|c|c|c|c|c|c|c|c|c|}
\hline \# & Target & $\begin{array}{l}\text { RA (2000) } \\
\text { hh:mm:ss }\end{array}$ & $\begin{array}{c}\text { Dec (2000) } \\
\text { dd:mm:ss }\end{array}$ & $\begin{array}{c}\text { Date } \\
\mathrm{dd} / \mathrm{mm} / \mathrm{yy}\end{array}$ & $\begin{array}{c}\text { UT } \\
\text { hh:mm }\end{array}$ & $\begin{array}{l}\mathrm{T} \\
\mathrm{m}\end{array}$ & $\overline{\bar{Q}}$ & $\begin{array}{l}\text { Seeing } \\
\text { arcsec }\end{array}$ & Calibrator \\
\hline 1 & UX Ori & 05:04:29.99 & $-03: 47: 14.3$ & $22 / 10 / 2003$ & $08: 38$ & 10 & $\mathrm{G}$ & $1.19 / 1.16$ & $\gamma$ Ori \\
\hline 2 & HD 34282 & 05:16:00.48 & $-09: 48: 35.4$ & $05 / 11 / 2003$ & $06: 15$ & 14 & G & $1.10 / 1.40$ & $\gamma$ Ori $^{\star}$ \\
\hline 3 & V346 Ori & $05: 24: 42.80$ & $+01: 43: 48.3$ & $05 / 11 / 2003$ & 07:13 & 6 & G & $1.53 / 1.49$ & $\gamma \mathrm{Ori}^{\star}$ \\
\hline 4 & CO Ori & $05: 27: 38.34$ & $+11: 25: 38.9$ & $28 / 10 / 2004$ & 04:37 & 8 & G & $0.44 / 0.63$ & HD 212330 \\
\hline 5 & HD 35929 & $05: 27: 42.79$ & $-08: 19: 38.4$ & $03 / 10 / 2004$ & $07: 38$ & 18 & G & $1.08 / 0.85$ & HD 38858 \\
\hline 6 & $\mathrm{StH} \alpha 41$ & $05: 29: 11.44$ & $-06: 08: 05.4$ & $03 / 10 / 2004$ & $08: 56$ & 8 & G & $1.08 / 1.08$ & HD 38858 \\
\hline 7 & HK Ori & 05:31:28.01 & $+12: 09: 10.7$ & $09 / 02 / 2004$ & $00: 36$ & 6 & $\mathrm{~J}$ & $1.17 / 0.84$ & $\gamma$ Ori \\
\hline 8 & HD 244604 & $05: 31: 57.25$ & $+11: 17: 41.4$ & $09 / 02 / 2004$ & 01:39 & 6 & G & $0.88 / 1.07$ & $\gamma$ Ori $^{\star}$ \\
\hline 9 & RY Ori & 05:32:09.96 & $-02: 49: 46.8$ & $04 / 10 / 2004$ & $06: 34$ & 18 & G & $0.57 / 0.60$ & HD 44594 \\
\hline 10 & $\mathrm{~T}$ Ori & $05: 35: 50.35$ & $-05: 28: 35.1$ & $05 / 11 / 2003$ & 07:39 & 6 & G & $1.72 / 1.66$ & $\gamma \mathrm{Ori}^{\star}$ \\
\hline 11 & V380 Ori & $05: 36: 25.43$ & $-06: 42: 57.7$ & $05 / 11 / 2003$ & 08:00 & 6 & G & $1.64 / 1.60$ & $\gamma \mathrm{Ori}^{\star}$ \\
\hline 12 & V586 Ori & 05:36:59.25 & $-06: 09: 16.4$ & $05 / 11 / 2003$ & 08:27 & 18 & G & $1.98 / 1.71$ & $\gamma \mathrm{Ori}^{\star}$ \\
\hline 13 & BF Ori & $05: 37: 13.26$ & $-06: 35: 00.6$ & 08/10/2004 & $06: 51$ & 18 & G & $1.46 / 1.64$ & $\gamma$ Ori \\
\hline 14 & HD 37357 & 05:37:47.08 & $-06: 42: 30.3$ & $08 / 10 / 2004$ & $07: 24$ & 14 & G & $1.56 / 1.89$ & $\gamma$ Ori \\
\hline 15 & N3sk 81 & 05:38:09.30 & $-06: 49: 17.0$ & 08/10/2004 & 09:06 & 14 & G & $1.69 / 1.86$ & $\gamma$ Ori \\
\hline 16 & HD 37411 & 05:38:14.51 & $-05: 25: 13.3$ & 08/10/2004 & 08:38 & 10 & G & $1.31 / 1.18$ & $\gamma$ Ori \\
\hline 17 & Haro 13a & 05:38:18.20 & $-07: 02: 26.6$ & $28 / 10 / 2004$ & 05:39 & 6 & G & $0.42 / 0.44$ & HD 38858 \\
\hline 18 & V599 Ori & 05:38:58.60 & $-07: 16: 46.0$ & 09/10/2004 & 08:36 & 12 & G & $0.61 / 0.76$ & $\epsilon \mathrm{Cen}^{\star}$ \\
\hline 19 & V350 Ori & 05:40:11.78 & $-09: 42: 11.4$ & 09/10/2004 & 05:05 & 18 & G & $1.13 / 1.48$ & $\epsilon \mathrm{Cen}^{\star}$ \\
\hline 20 & HD 37806 & 05:41:02.29 & $-02: 43: 00.7$ & $28 / 10 / 2004$ & $06: 46$ & 6 & G & $0.48 / 0.45$ & HD 38858 \\
\hline 21 & HD 250550 & 06:01:59.99 & $+16: 30: 56.7$ & $12 / 01 / 2005$ & $04: 29$ & 6 & G & $1.78 / 1.75$ & $\gamma$ Ori \\
\hline 22 & VY Mon & $06: 31: 06.90$ & $+10: 26: 05.3$ & $12 / 01 / 2005$ & 04:50 & 4 & G & $2.08 / 2.00$ & $\gamma$ Ori \\
\hline \multirow[t]{2}{*}{23} & $\mathrm{LkH} \alpha 215$ & $06: 32: 41.80$ & $+10: 09: 33.6$ & $21 / 01 / 2005$ & 01:55 & 8 & G & $0.76 / 0.70$ & $\xi$ Ori \\
\hline & & & & $26 / 02 / 2005$ & 02:08 & 8 & & $1.20 / 1.42$ & $\rho$ Cen \\
\hline \multirow[t]{2}{*}{24} & HD 259431 & 06:33:05.19 & $+10: 19: 20.0$ & $21 / 01 / 2005$ & $02: 54$ & 6 & G & $0.88 / 0.79$ & $\lambda$ Lep \\
\hline & & & & $25 / 02 / 2005$ & 03:42 & 6 & & $0.67 / 0.57$ & $\epsilon$ Cen \\
\hline 25 & R Mon & 06:39:09.89 & $+08: 44: 10.0$ & $10 / 01 / 2005$ & $05: 32$ & 6 & G & $0.96 / 0.72$ & $\gamma$ Ori \\
\hline 26 & V590 Mon & $06: 40: 44.57$ & $+09: 48: 02.1$ & $10 / 01 / 2005$ & 03:46 & 28 & G & $0.73 / 1.94$ & $\gamma$ Ori \\
\hline 27 & GU CMa & 07:01:49.51 & $-11: 18: 03.3$ & $01 / 03 / 2004$ & 03:48 & 6 & $\mathrm{~J}$ & $0.66 / 0.84$ & $\epsilon$ Cen \\
\hline \multirow[t]{2}{*}{28} & HD 53367 & $07: 04: 25.53$ & $-10: 27: 15.7$ & $12 / 02 / 2005$ & $05: 45$ & 6 & G & $1.00 / 0.76$ & $\beta \mathrm{Cir}$ \\
\hline & & & & $19 / 11 / 2003$ & $08: 15$ & 6 & & $0.50 / 0.51$ & $\gamma \mathrm{Ori}^{\star}$ \\
\hline 29 & NX Pup & $07: 19: 28.26$ & $-44: 35: 11.3$ & $09 / 02 / 2004$ & 05:04 & 6 & G & $0.81 / 0.85$ & $\gamma$ Ori \\
\hline 30 & HD 76534 & 08:55:08.71 & $-43: 27: 59.9$ & $11 / 01 / 2005$ & 04:54 & 18 & G & $2.03 / 1.85$ & $v$ Pup \\
\hline 31 & HD 85567 & 09:50:28.54 & $-60: 58: 03.0$ & $20 / 01 / 2005$ & 09:06 & 6 & G & $0.64 / 0.59$ & $\pi \mathrm{Sco}$ \\
\hline 32 & HD 95881 & $11: 01: 57.62$ & $-71: 30: 48.4$ & $23 / 01 / 2005$ & 05:34 & 6 & G & $0.81 / 0.76$ & $\xi^{2} \mathrm{Cen}$ \\
\hline 33 & HD 97048 & 11:08:03.32 & $-77: 39: 17.5$ & $18 / 07 / 2004$ & 01:01 & 6 & G & $0.55 / 0.63$ & $\epsilon \mathrm{Cen}^{\star}$ \\
\hline 34 & HD 100546 & $11: 33: 25.44$ & $-70: 11: 41.2$ & $27 / 07 / 2004$ & 23:49 & 6 & G & $1.24 / 1.41$ & $\kappa$ Eri \\
\hline 35 & HD 101412 & $11: 39: 44.46$ & $-60: 10: 27.7$ & $28 / 07 / 2004$ & $00: 20$ & 8 & G & $1.33 / 1.29$ & $\kappa$ Eri \\
\hline 36 & T Cha & $11: 57: 13.53$ & $-79: 21: 31.5$ & $18 / 07 / 2004$ & 01:26 & 10 & $\mathrm{~J}$ & $0.88 / 0.71$ & $\epsilon \mathrm{Cen}^{\star}$ \\
\hline 37 & LSS 3027 B & 13:19:03.98 & $-62: 34: 10.1$ & $10 / 05 / 2004$ & 05:39 & 8 & $\mathrm{~L}$ & $0.78 / 0.90$ & $\epsilon$ Cen \\
\hline 38 & HD 130437 & $14: 50: 50.26$ & $-60: 17: 10.3$ & $10 / 05 / 2004$ & 06:03 & 8 & G & $0.93 / 0.84$ & $\epsilon$ Cen \\
\hline 39 & SS73 44 & $15: 03: 23.80$ & $-63: 22: 59.0$ & $05 / 09 / 2003$ & 01:08 & 8 & G & $0.83 / 0.95$ & $\kappa \mathrm{Cet}^{\star}$ \\
\hline 40 & HD 132947 & 15:04:56.05 & $-63: 07: 52.6$ & $05 / 09 / 2003$ & 01:35 & 8 & $\mathrm{~L}+\mathrm{J}$ & $0.88 / 0.93$ & $\kappa \mathrm{Cet}^{\star}$ \\
\hline 41 & HD 141569 & $15: 49: 57.75$ & $-03: 55: 16.4$ & $22 / 08 / 2004$ & $23: 15$ & 8 & $\mathrm{~J}$ & $0.72 / 0.73$ & $\sigma \mathrm{Sco}$ \\
\hline 42 & HD 142666 & 15:56:40.02 & $-22: 01: 40.0$ & $23 / 08 / 2004$ & 00:53 & 6 & $\mathrm{~J}$ & $0.96 / 0.96$ & $\sigma \mathrm{Sco}$ \\
\hline 43 & HD 142527 & $15: 56: 41.89$ & $-42: 19: 23.3$ & $05 / 09 / 2003$ & 02:05 & 6 & G & $1.32 / 0.91$ & $\kappa \mathrm{Cet}^{\star}$ \\
\hline 44 & HR 5999 & $16: 08: 34.29$ & $-39: 06: 18.3$ & $22 / 07 / 2004$ & 03:46 & 6 & $\mathrm{~J}$ & $1.61 / 1.82$ & HD 172910 \\
\hline 45 & HD 150193 & $16: 40: 17.92$ & $-23: 53: 45.2$ & $10 / 05 / 2004$ & 06:50 & 6 & G & $0.83 / 0.86$ & $\kappa \mathrm{Aql}$ \\
\hline 46 & AK Sco & $16: 54: 44.85$ & $-36: 53: 18.6$ & $10 / 05 / 2004$ & $07: 15$ & 8 & G & $0.85 / 0.89$ & $\kappa$ Aql \\
\hline 47 & V921 Sco & $16: 59: 06.90$ & $-42: 42: 08.0$ & $22 / 07 / 2004$ & 03:01 & 6 & G & $1.76 / 1.82$ & HD 172910 \\
\hline 48 & KK Oph & 17:10:08.07 & $-27: 15: 18.2$ & $22 / 07 / 2004$ & 03:26 & 8 & $\mathrm{~J}$ & $1.39 / 1.36$ & HD 172910 \\
\hline 49 & HD 163296 & $17: 56: 21.29$ & $-21: 57: 21.9$ & $19 / 09 / 2003$ & 02:07 & 6 & G & $1.54 / 1.74$ & $\kappa$ Cet \\
\hline 50 & MWC 297 & $18: 27: 39.60$ & $-03: 49: 52.0$ & $19 / 09 / 2003$ & $02: 27$ & 4 & G & $1.67 / 1.64$ & $\kappa$ Cet \\
\hline 51 & VV Ser & $18: 28: 47.87$ & $+00: 08: 39.6$ & $19 / 09 / 2003$ & 02:46 & 6 & G & $1.42 / 1.32$ & $\kappa$ Cet \\
\hline
\end{tabular}


B. Acke \& M. E. van den Ancker: Diamonds in Herbig Ae/Be systems, Online Material p 6

Table 4. continued.

\begin{tabular}{clrccccccc}
\hline \hline$\#$ & Target & $\begin{array}{r}\text { RA (2000) } \\
\text { hh:mm:ss }\end{array}$ & $\begin{array}{c}\text { Dec (2000) } \\
\text { dd:mm:ss }\end{array}$ & $\begin{array}{c}\text { Date } \\
\text { dd/mm/yy }\end{array}$ & $\begin{array}{c}\text { UT } \\
\text { hh:mm }\end{array}$ & $\begin{array}{c}\text { T } \\
\text { m }\end{array}$ & $\begin{array}{c}\text { Q } \\
\text { Seeing } \\
\text { arcsec }\end{array}$ & Calibrator \\
\hline 52 & MWC 300 & $18: 29: 25.69$ & $-06: 04: 37.3$ & $19 / 09 / 2003$ & $03: 02$ & 6 & $\mathrm{G}$ & $1.42 / 1.45$ & $\kappa$ Cet \\
53 & AS 310 & $18: 33: 21.17$ & $-04: 58: 06.7$ & $19 / 09 / 2003$ & $03: 26$ & 14 & $\mathrm{~L}$ & $1.57 / 1.28$ & $\kappa$ Cet \\
54 & S CrA & $19: 01: 08.60$ & $-36: 57: 20.0$ & $19 / 09 / 2003$ & $03: 48$ & 8 & $\mathrm{G}$ & $1.49 / 1.39$ & $\kappa$ Cet \\
55 & TY CrA & $19: 01: 40.83$ & $-36: 52: 33.9$ & $19 / 09 / 2003$ & $04: 08$ & 8 & $\mathrm{G}$ & $1.14 / 1.04$ & $\kappa$ Cet \\
56 & R CrA & $19: 01: 53.65$ & $-36: 57: 07.6$ & $19 / 09 / 2003$ & $04: 27$ & 6 & $\mathrm{G}$ & $0.83 / 0.91$ & $\kappa$ Cet \\
57 & T CrA & $19: 01: 58.80$ & $-36: 57: 49.0$ & $26 / 09 / 2003$ & $01: 38$ & 8 & $\mathrm{G}$ & $0.99 / 1.24$ & BY Cap \\
58 & HD 179218 & $19: 11: 11.25$ & $+15: 47: 15.6$ & $01 / 10 / 2003$ & $02: 01$ & 6 & $\mathrm{G}$ & $0.63 / 0.66$ & HD 9562 \\
59 & HBC 687 & $19: 29: 00.80$ & $+09: 38: 43.0$ & $26 / 09 / 2003$ & $02: 08$ & 20 & $\mathrm{~L}$ & $1.11 / 1.12$ & HD 9562 \\
60 & V1295 Aql & $20: 03: 02.52$ & $+05: 44: 16.7$ & $19 / 09 / 2003$ & $04: 46$ & 6 & $\mathrm{G}$ & $0.78 / 0.90$ & $\kappa$ Cet \\
\hline
\end{tabular}




\section{Appendix A}

Apart from HD 97048 and MWC 297, a few other targets display a feature in the 3.5 micron region. Due to their low feature/continuum ratio, however, it is difficult to discern them from data reduction artefacts. In this Appendix we will try to clarify which features are astrophysical, and which are not.

In our opinion, the main source of data reduction artefacts is the correction procedure for atmospheric absorption lines. Assume that $S_{\lambda}$ is the flux emitted by the standard star (STD), and $\tau_{\lambda}$ the atmospheric optical depth at a certain airmass and at the time of the STD observation. Define the scientific target's flux as $T_{\lambda}$ and the corresponding atmospheric optical depth as $\sigma_{\lambda}$. We assume that the airmasses at the time of both observations are the same. Note however that even in this case $\tau_{\lambda}$ and $\sigma_{\lambda}$ are not necessarily the same, since the atmospheric conditions (e.g. air density, humidity) may differ between the two observations. The STD's flux can be approximated by a photospheric model. If $F_{\lambda}$ is the measured STD flux, then $\mathrm{e}^{\tau_{\lambda}}=S_{\lambda \text {,model }} / F_{\lambda}$ can be estimated. This atmospheric correction factor is then applied to the target measurement, which leads to an estimate of the target's flux, $\widetilde{T}_{\lambda}=\left(T_{\lambda} \mathrm{e}^{-\sigma_{\lambda}}\right) \mathrm{e}^{\tau_{\lambda}}=T_{\lambda} \mathrm{e}^{\left(\tau_{\lambda}-\sigma_{\lambda}\right)} \approx T_{\lambda}$. Slight differences between $\tau_{\lambda}$ and $\sigma_{\lambda}$ introduce artefacts in the reduced spectrum of the science target. As a first order approach, we assume that the main difference in telluric extinction occurs due to a geometrical and/or atmospheric density effect. Such an effect is linear in $\tau_{\lambda}$. Hence $\mathrm{e}^{\left(\tau_{\lambda}-\sigma_{\lambda}\right)}$ is proportional to $\mathrm{e}^{C \tau_{\lambda}}$, with $C$ a constant. The bottom two spectra in Fig. A.1 show the influence of over- and undercorrection on a flat continuum. The artefacts that are relevant in this discussion are the "emission features" at $3.505 \mu \mathrm{m}$ in the case of undercorrection $\left(\propto \mathrm{e}^{-\tau_{\lambda}}\right)$, and $3.54 \mu \mathrm{m}$ in the case of overcorrection $\left(\propto \mathrm{e}^{\tau_{\lambda}}\right)$.

We will try to separate the real spectral features from the artefacts in the 14 candidate nanodiamond emitters listed in Table 5. Two of them, HD 97048 and MWC 297, are known to display the $3.53 \mu \mathrm{m}$ feature and will serve as a reference. The vast majority of the other 12 detected features is most likely due to the data reduction procedure. In Fig. A.1, the normalized spectra of the candidate nanodiamond emitters have been displayed. We have indicated which spectra appear to be over- and undercorrected. Most of the features follow the spectral shape of $\mathrm{e}^{\tau_{\lambda}}$ or $\mathrm{e}^{-\tau_{\lambda}}$. Only for V921 Sco, HD 163296 and T CrA, the detected feature's appearance deviates from the artefacts' shape. Even in these cases however, it remains difficult to exclude an artefactual origin. An indication may come from the upper limit to the $3.53 \mu \mathrm{m}$ line fluxes measured in the ISO spectrum. The upper limits for HD 163296 and T CrA are in agreement with the measured ISAAC line fluxes $(<2.5$ and $<1.4$ vs. 2.3 and $0.65 \times$ $10^{-15} \mathrm{~W} \mathrm{~m}^{-2}$ ). For V921 Sco, the observed line flux exceeds the ISO upper limit by a factor three (<3.7 vs. $\left.10.3 \times 10^{-15} \mathrm{~W} \mathrm{~m}^{-2}\right)$, which makes the NANO $3.53 \mu \mathrm{m}$ detection in this source rather suspicious. In the paper, V921 Sco, HD 163296 and T CrA are nevertheless treated as the remaining three candidate nanodiamond emitters.

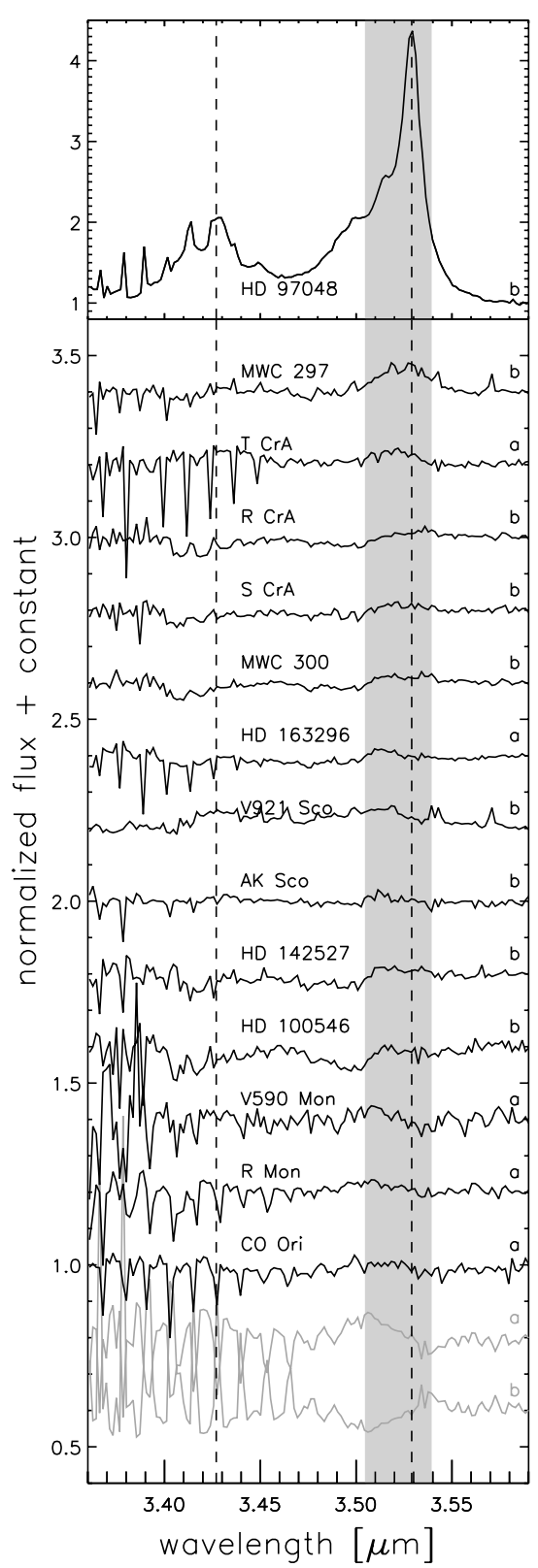

Fig. A.1. The normalized spectra of the candidate nanodiamond emitters. The bottom two spectra are a standard-star spectrum, and the inverse of it. They represent the spectral shape of an undercorrected continuum $\mathrm{e}^{-\tau_{\lambda}}$ (a) and an overcorrected continuum $\mathrm{e}^{\tau_{\lambda}}$ (b). The peak position of the two curves are indicated by the edges of the grey box. The target spectra are flagged with a and b, based on the apparent shape of the continuum. The real detected nanodiamond features in MWC 297 and HD 97048 are plotted at the top of the figure. The latter is drawn in a separate box because of its impressive strength compared to the features or artefacts in the other spectra. The peak position of the 3.53 and $3.43 \mu \mathrm{m}$ features in this spectrum have been indicated by the dashed line. Most of the detected features can be brought back to data reduction artefacts. Only the features in V921 Sco, HD 163296 and T CrA may have deviating shapes. 